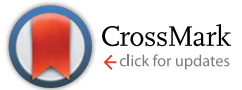

Cite this: RSC Adv., 2015, 5, 89731

Received 11th September 2015 Accepted 13th October 2015

DOI: $10.1039 / \mathrm{c} 5 \mathrm{ra} 18649 \mathrm{~h}$

www.rsc.org/advances

\section{Liquid crystalline dihydroazulene photoswitches $\uparrow$}

\author{
Anne Ugleholdt Petersen, ${ }^{a}$ Martyn Jevric, ${ }^{a}$ Richard J. Mandle, ${ }^{\mathrm{b}}$ Edward J. Davis, ${ }^{\mathrm{b}}$

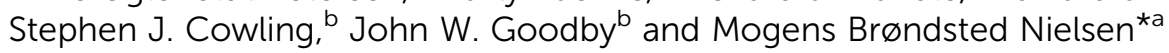

\begin{abstract}
A large selection of photochromic dihydroazulene (DHA) molecules incorporating various substituents at position 2 of the DHA core was prepared and investigated for their ability to form liquid crystalline phases. Incorporation of an octyloxy-substituted biphenyl substituent resulted in nematic phase behavior and it was possible to convert one such compound partly into its vinylheptafulvene (VHF) isomer upon irradiation with light when in the liquid crystalline phase. This conversion resulted in an increase in the molecular alignment of the phase. In time, the meta-stable VHF returns to the DHA where the alignment is maintained. The systematic structural variation has revealed that a biaryl spacer between the DHA and the alkyl chain is needed for liquid crystallinity and that the one aromatic ring in the spacer cannot be substituted by a triazole. This work presents an important step towards employing the dihydroazulenevinylheptafulvene photo/thermoswitch in photoactive liquid crystalline materials.
\end{abstract}

\section{Introduction}

In the field of liquid crystals, it has been a goal to control the phases and order with an external stimulus. Some of the stimuli which have been applied include temperature, magnetic field, electric field and light. ${ }^{1}$ Light is an interesting stimulus, since this gives the possibility for remote control. Indeed, it has found use in image storing devices. ${ }^{2,3}$ Specifically, this has been shown with azobenzene polymer matrix, where an image was stored for 8 months without significant decay. ${ }^{2}$ Other photochromic molecules such as dithienylethenes, spiropyrans and fulgides have also shown suitable properties for this kind of application. ${ }^{4-6}$ Here we present a study on liquid crystalline systems based upon the dihydroazulene (DHA) photoswitch (Scheme 1). When irradiated with light, DHA 1 undergoes a ring-opening to the vinylheptafulvene (VHF) 2, which can then close back to the initial DHA via a thermal electrocyclization reaction. ${ }^{7}$

The main advantage of the DHA-VHF photoswitch system is that only the DHA to VHF conversion is light-induced, which
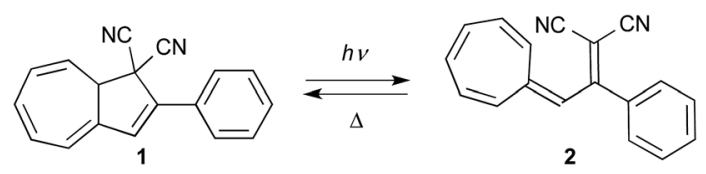

Scheme 1 DHA 1/VHF 2 system.

\footnotetext{
${ }^{a}$ Department of Chemistry, University of Copenhagen, Universitetsparken 5, DK-2100 Copenhagen Ø, Denmark.E-mail: mbn@chem.ku.dk

${ }^{b}$ Department of Chemistry, University of York, Heslington, York, YO10 5DD, UK

$\dagger$ Electronic supplementary information (ESI) available. See DOI: 10.1039/c5ra18649h
}

means that a broad spectrum of light can be used without triggering the VHF to DHA back-reaction. The DHA ringopening is associated with significant changes in the physical properties of the corresponding VHF. Thus, ring-opening is associated with an increase in dipole moment and a change in color from yellow (DHA) to intense red (VHF). ${ }^{8,9}$ We became interested to elucidate if this difference in properties could give rise to reversible, photochemically induced phase transitions.

Molecules with liquid crystalline properties can have differing constructions, but for the purpose of this work, attention was given to rod-like structures. Typically, rod-like, liquid crystalline structures have two aliphatic chains extending from a rigid 'core' unit such as biphenyl or one alkyl chain with an additional terminal substituent such as a nitrile. ${ }^{10}$ It was decided to incorporate either a cyanophenyl-substituted alkyl in the one end of DHA or an octyl chain. In the literature there are many examples of the cyanobiphenyl being used as the terminal substituent, ${ }^{\mathbf{1 1}}$ but as this unit has absorption properties which conflict with the wavelength needed for the ringopening event of DHA, the cyanophenyl substituent was used predominantly in this study, as in structures 3a-e and $4 \mathbf{4 a - e}$ (Fig. 1). Structures $\mathbf{3 f}-\mathbf{i}$ and $\mathbf{4 j}$ instead have an octyl end-group. Both cyanophenyl and octyl groups have been used previously to achieve liquid crystallinity. ${ }^{\mathbf{1 2}}$

\section{Results and discussion}

\subsection{Synthesis}

The most convenient method for the construction of 2substituted DHAs originates from derivatized acetophenones. Several strategies were undertaken in the pursuit of obtaining the starting acetophenones (Scheme 2). From the simple precursors 5a-e, 6, 7a-e, 8, acetophenones 9a-e were prepared 
<smiles>N#Cc1ccc(OCCOc2ccc(C3=CC4=CC=CC=CC4C3(C#N)C#N)cc2)cc1</smiles><smiles>[R10]Oc1ccc(-c2ccc(C3=CC4=CC=CC=CC4C3(C)C#N)cc2)c([X])c1[X]</smiles><smiles>COc1ccc(C2=CC3=CC=CC=CC3C2(C#N)C#N)cc1</smiles><smiles>CC1(N)C(c2ccc(-c3cn(CCOc4ccc(C#N)cc4)nn3)cc2)=CC2=CC=CC=CC21</smiles>

Fig. 1 DHAs employed in this study.

in two successive reactions. Thus, dibromoalkanes $5 \mathbf{a}-\mathbf{e}$ of various chain lengths were treated with phenols 6 and 8 . Conversely, acetophenones containing biaryl units, $\mathbf{9 f}$ and $\mathbf{9 g}$, were obtained in good yields from a palladium-catalyzed Suzuki cross-coupling reaction from 4-iodoacetophenone $\mathbf{1 0}$ with boronic acids 11f and 11g, respectively, using conditions that proved fruitful in the synthesis of the benzaldehyde analogues. ${ }^{13}$ Finally, $9 \mathbf{h}$ could be formed in high yield by a Friedel-Crafts reaction of $\mathbf{1 2}$ with acetyl chloride using a literature procedure. ${ }^{14}$ Compound $\mathbf{9 i}$ was purchased.

Acetophenones $\mathbf{9 a - i}$ could be transformed successfully into the corresponding DHAs 3a-i (Scheme 3) by firstly converting the carbonyl moiety into a crotononitrile unit in a Knoevenagel condensation furnishing intermediates 13a-i, generally in good yields. Intermediates 13a-i were then treated with tropylium tetrafluoroborate in the presence of triethylamine to affix the cycloheptatriene unit to the malonitriles which could be further converted into $\mathbf{3 a}-\mathbf{i}$ by subsequent oxidation using tritylium tetrafluoroborate followed by treatment with triethylamine. Alternatively, biaryl DHAs $\mathbf{3 f}$ and $\mathbf{3 g}$ could be obtained directly from DHA 15 in a Suzuki reaction with boronic acids 11 and $\mathbf{1 1 g}$, using the catalytic system of palladium acetate and RuPhos (Scheme 4). A separate strategy involving the introduction of the extended chains could also be effected using the coppercatalyzed azide-alkyne cycloaddition (CuAAC) ${ }^{\mathbf{1 5}}$ to form triazole compounds. Triazoles have previously been incorporated successfully into liquid crystalline compounds ${ }^{\mathbf{1 6}}$ and, synthetically, DHA has been found to withstand the conditions. ${ }^{17}$ Alkyl bromides $7 \mathbf{a}-\mathbf{e}$ were converted into their corresponding azides 14a-e (Scheme 5) and when subjected to treatment with 10 mol\% cuprous iodide in the presence of $\mathbf{1 6}$ and Hünig's base, triazoles $\mathbf{4 a - e}$ were achieved. DHA $\mathbf{4 j}$ could be made from a CuAAC of octyl azide with 16 in the same manner.
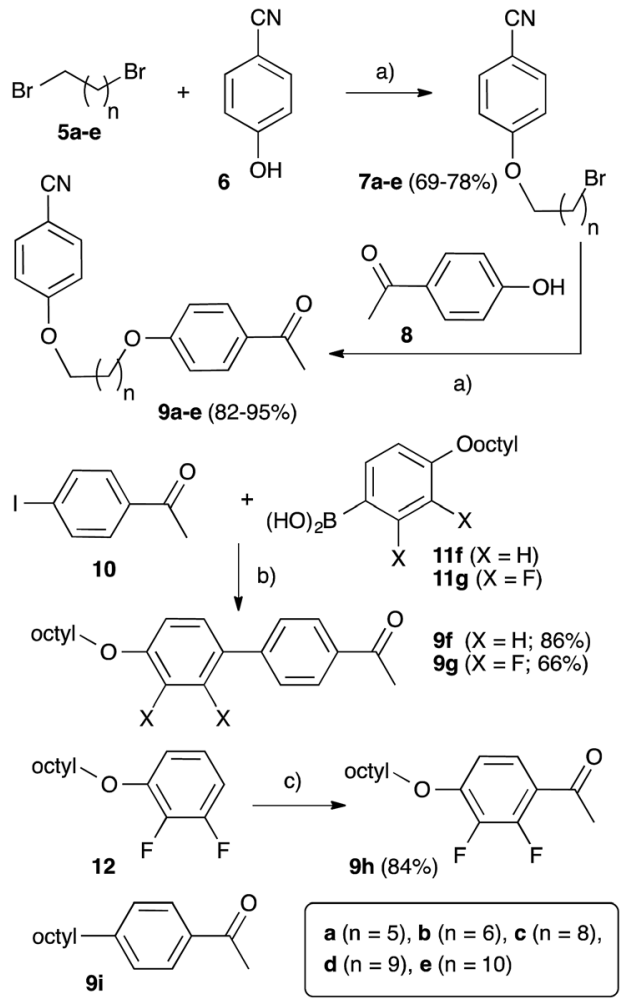

Scheme 2 Synthesis of acetophenone starting materials. Conditions: (a) $\mathrm{K}_{2} \mathrm{CO}_{3}$, acetone, reflux; (b) $5 \% \mathrm{Pd}\left(\mathrm{PPh}_{3}\right)_{4}, \mathrm{~K}_{3} \mathrm{PO}_{4}$, toluene $/ \mathrm{H}_{2} \mathrm{O}$, rt to $80{ }^{\circ} \mathrm{C}$; (c) $\mathrm{FeCl}_{3}, \mathrm{AcCl}, \mathrm{CH}_{2} \mathrm{Cl}_{2}, 0^{\circ} \mathrm{C}$ to rt.
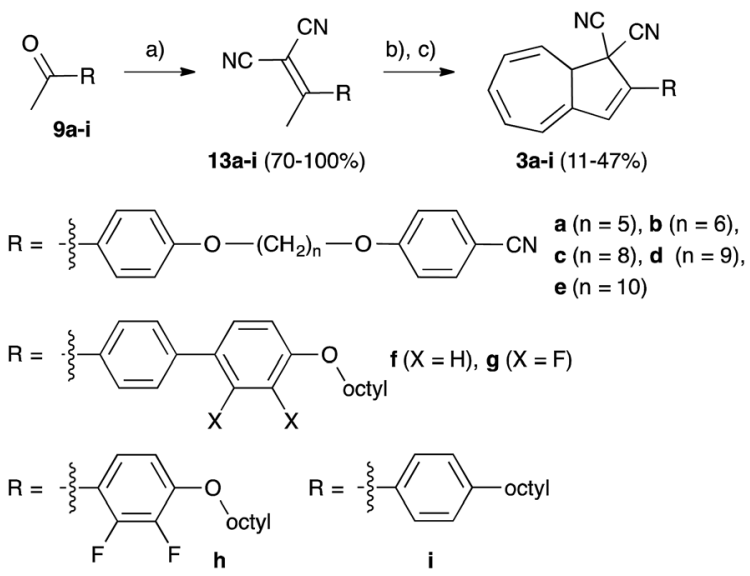

Scheme 3 Synthesis of DHAs. Conditions: (a) $\mathrm{CH}_{2}(\mathrm{CN})_{2}, \mathrm{NH}_{4} \mathrm{OAC}$, toluene, $\mathrm{AcOH}, \Delta$. (b) $\left[\mathrm{C}_{7} \mathrm{H}_{7}\right] \mathrm{BF}_{4}, \mathrm{NEt}_{3}, \mathrm{CH}_{2} \mathrm{Cl}_{2},-78^{\circ} \mathrm{C}$. (c) $\left[\mathrm{Ph}_{3} \mathrm{C}_{3} \mathrm{BF}_{4}\right.$, $\mathrm{CH}_{2} \mathrm{ClCH}_{2} \mathrm{Cl}, \Delta$, then toluene, $\mathrm{NEt}_{3}, 0^{\circ} \mathrm{C}$.

We also decided to prepare the corresponding VHFs of two of the DHAs (to study their liquid crystalline properties of relevance for the switching studies, vide infra). It has been shown that DHA can be ring-opened by treatment with $\mathrm{AlCl}_{3}$ to afford the corresponding $\mathrm{VHF}^{\mathbf{1 8}}$ and this method could be used to convert 3f into 17 in good yield (Scheme 6). When the method was used on $\mathbf{3 g}$, not only did the DHA undergo ring-opening to 


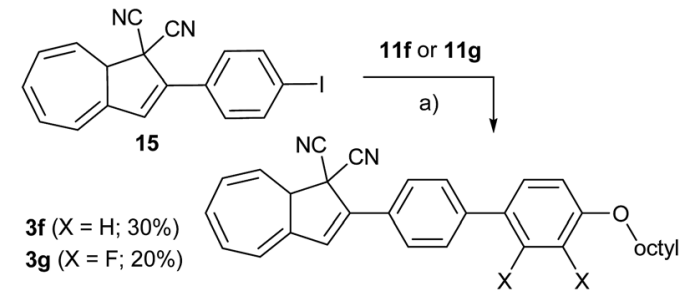

Scheme 4 Functionalization of DHA via Suzuki cross-coupling Conditions: (a) $\mathrm{Pd}(\mathrm{OAc})_{2}$, RuPhos, $\mathrm{K}_{3} \mathrm{PO}_{4}$, toluene $/ \mathrm{H}_{2} \mathrm{O}$. RuPhos $=2$ dicyclohexylphosphino-2', $6^{\prime}$-diisopropoxybiphenyl.
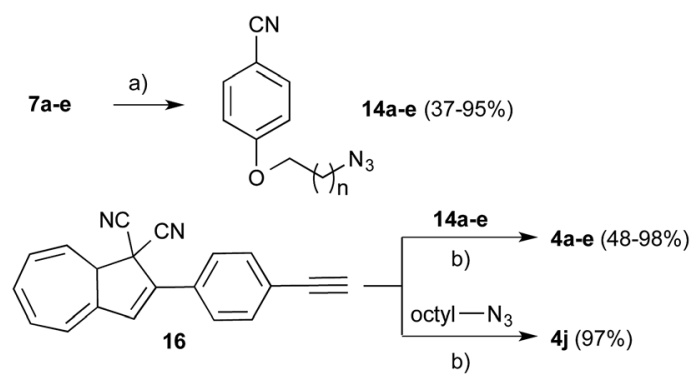

Scheme 5 Functionalization of DHA via CuAAC. Conditions: (a) $\mathrm{NaN}_{3}$, DMSO, $50^{\circ} \mathrm{C}$; (b) Cul, Hünig's base, toluene.<smiles>CC(=O)OOc1ccc(-c2ccc(C(C=C3C=CC=CC=C3)=C(C)C#N)cc2)cc1</smiles><smiles>CC(C#N)=C(C=C1C=CC=CC=C1)c1ccc(-c2ccc(O)c(F)c2F)cc1</smiles>

Scheme 6 Synthesis of VHFs. Conditions (a) $\mathrm{AlCl}_{3}, \mathrm{CH}_{2} \mathrm{Cl}_{2}, 0{ }^{\circ} \mathrm{C} / \mathrm{rt}$, then $\mathrm{H}_{2} \mathrm{O}, \mathrm{O}^{\circ} \mathrm{C}$.

the VHF, but these reaction conditions also resulted in the cleavage of the octyl chain to form phenol 18.

\subsection{Photochemical properties}

All DHAs made in this study show photoactive properties akin to that of unfunctionalized DHA 1. All DHAs exhibited an absorption maximum at around $360 \mathrm{~nm}$ and when irradiated with light of this wavelength, they undergo an electrocyclic ringopening reaction. Thereby a peak at $475 \mathrm{~nm}$ emerges, corresponding to the formation of the VHF (Table 1). The rate of VHF to DHA thermal ring-closure has been found to be influenced greatly by the introduction of electron-donating/accepting functional groups. ${ }^{9,19}$ The VHFs of DHAs 3a-e all exhibit a rate constant corresponding to that of the $p$-methoxy substituted derivative of DHA 1. The rate of ring-closure for the VHFs of the triazole derivatives $\mathbf{4 a}-\mathbf{e}$ and $\mathbf{4 j}$ show a small deviation from
Table 1 Characteristic absorption maxima and kinetics data for VHF to $\mathrm{DHA}$ conversions in acetonitrile at $25^{\circ} \mathrm{C}$

\begin{tabular}{lllll}
\hline & $\begin{array}{l}\text { DHA, } \lambda_{\max } \\
(\mathrm{nm})\end{array}$ & $\begin{array}{l}\mathrm{VHF}, \lambda_{\max } \\
(\mathrm{nm})\end{array}$ & $\begin{array}{l}\mathrm{VHF} \rightarrow \mathrm{DHA}, k \\
\left(10^{-5} \mathrm{~s}^{-1}\right)\end{array}$ & $\begin{array}{l}\mathrm{VHF} \rightarrow \mathrm{DHA}, t_{1 / 2} \\
(\mathrm{~min})\end{array}$ \\
\hline 3a & 365 & 469 & 5.04 & 229 \\
3b & 366 & 470 & 5.00 & 231 \\
3c & 367 & 471 & 4.97 & 232 \\
3d & 366 & 470 & 4.97 & 232 \\
3e & 365 & 470 & 4.97 & 232 \\
$\mathbf{3 f}$ & 374 & 474 & 6.78 & 170 \\
$\mathbf{3 g}$ & 366 & 475 & 7.30 & 158 \\
$\mathbf{3 h}$ & 359 & 480 & 5.00 & 231 \\
$\mathbf{3 i}$ & 358 & 471 & 4.98 & 232 \\
$\mathbf{4 a}$ & 370 & 475 & 6.65 & 174 \\
$\mathbf{4 b}$ & 370 & 476 & 6.72 & 172 \\
$\mathbf{4 c}$ & 370 & 475 & 6.72 & 172 \\
$\mathbf{4 d}$ & 368 & 475 & 6.68 & 173 \\
$\mathbf{4 e}$ & 370 & 475 & 6.66 & 174 \\
$\mathbf{4 j}$ & 369 & 475 & 6.63 & 174
\end{tabular}

a formerly reported triazole functionalized DHA, ${ }^{17}$ which had a rate constant for the VHF ring-closure of $7.45 \times 10^{-5} \mathrm{~s}^{-1}$. The VHFs of $3 \mathbf{h}$ and $3 \mathbf{i}$ have comparable ring-closure rate constants as those of the VHFs of 3a-e and of formerly reported DHA with a $p$-methyl substituent. Inclusion of fluorine seems to have no effect for the VHF of $3 \mathbf{h}$, or its effect is cancelled by the electrondonating octyloxy substituent. For the biphenyls $\mathbf{3 f}$ and $\mathbf{3 g}$ the rate of VHF to DHA conversion is higher and more similar to that of 4a-e. Here the effect of the fluorine is clear; the rate increases to $7.30 \times 10^{-5} \mathrm{~s}^{-1}$ compared to the non-fluorinated compound with a rate of $6.78 \times 10^{-5} \mathrm{~s}^{-1}$.

\subsection{Physical properties}

The synthesized molecules were examined by polarized optical microscopy (POM), but all starting materials en route to and including DHAs 3a-e from the first two approaches did not show any liquid-crystalline properties. The same could also be said for DHAs $\mathbf{4 a - e}$ and $\mathbf{4 j}$. Instead, biaryl containing acetophenones 9 f and $\mathbf{9 g}$ and the corresponding DHAs $\mathbf{3 f}$ and $\mathbf{3 g}$ exhibited a liquid-crystalline phase and were examined by both POM and differential scanning calorimetry (DSC). Compound 9f was found to exhibit a mesophase. Indeed, 9f (Fig. 2) has been previously reported with two different transitions, where in one, it exhibited a smectic $\mathrm{E}$ phase,$^{20}$ and in the other, a melt was reported at $115-118{ }^{\circ} \mathrm{C}^{21}$ We found that under the microscope 9 f did exhibit a smectic phase (see Fig. 2), in agreement with the former report although definitive phase assignment was not possible due to the short range of supercooling. Hence, we denote it smectic X. Fig. 3 shows the DSC thermogram.

DSC showed a transition for a mesophase, where the size of the associated enthalpy to the phase transition is indicative of the formation of a smectic mesophase. The partially fluorinated analogue $9 \mathrm{~g}$ gave a lower melt and did not exhibit a liquidcrystalline phase during the heating process. Upon cooling, a peak corresponding to a liquid-crystalline phase could only be observed in the DSC at $53.8^{\circ} \mathrm{C}$. It was only possible to observe 


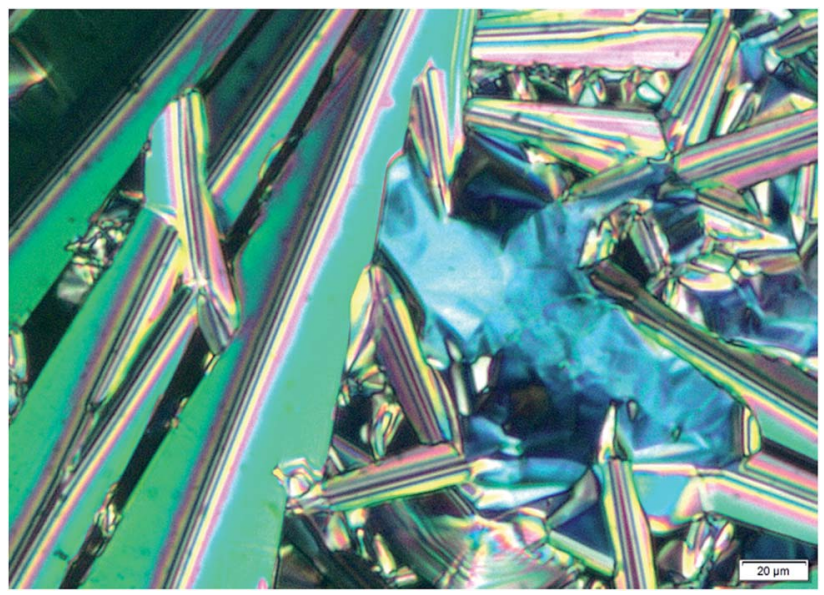

Fig. 2 Photomicrographs of the smectic $X$ phase of $9 f$ at $118^{\circ} \mathrm{C}$.
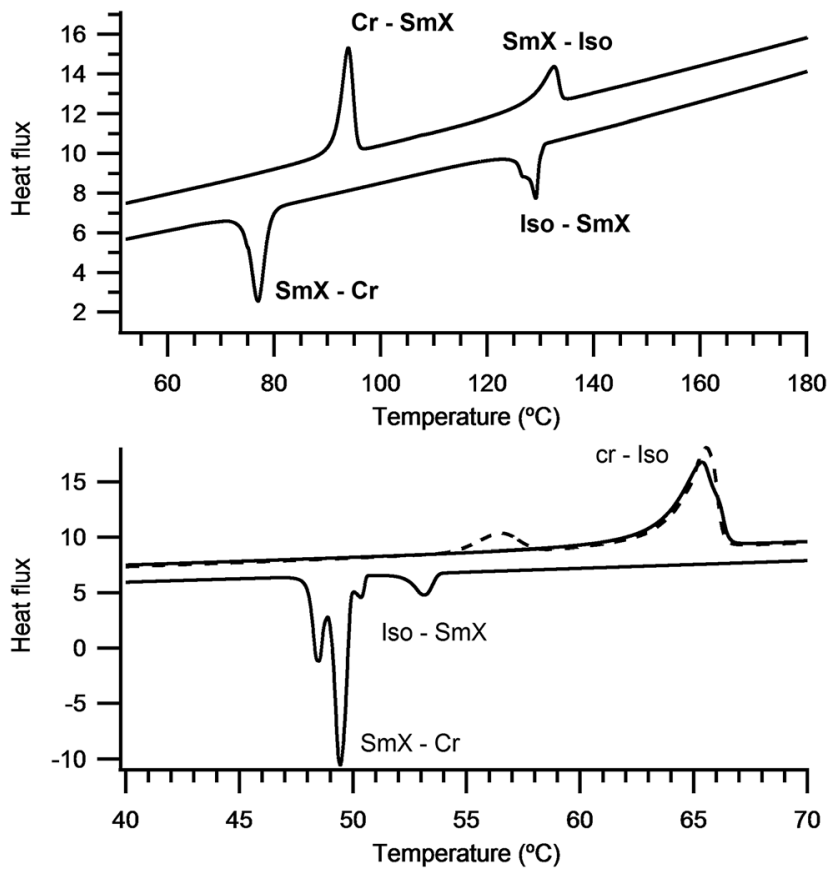

Fig. 3 (Top) DSC thermograms at a heating-cooling rate of $10{ }^{\circ} \mathrm{C}$ $\mathrm{min}^{-1}$ for compound 9f. (Bottom) DSC thermograms at a heatingcooling rate of $5{ }^{\circ} \mathrm{C} \mathrm{min}^{-1}$ for compound $9 \mathrm{~g}$, where the dashed line is the first heating.

this phase when the sample had been heated to $100{ }^{\circ} \mathrm{C}$ prior to cooling. When compound $9 \mathrm{~g}$ was heated to a lesser extent, such as 67 or $80^{\circ} \mathrm{C}$, the sample underwent crystallization during the cooling phase prior to the temperature where this initial phase transition was observed. When examined by POM, the crystallization occurred prior to the liquid crystal phase transition temperature. Table 2 lists transition temperatures for $\mathbf{9 f}$ and $\mathbf{9 g}$ and Table 3 the enthalpies and entropies associated with the transitions.

The two DHAs $3 \mathbf{f}$ and $\mathbf{3 g}$ showed liquid crystalline properties, whilst $\mathbf{3 h}$ and $\mathbf{3 i}$ did not exhibit any mesophases. This suggests that the additional aryl ring is important for the modified DHAs
Table 2 Transition temperatures $\left({ }^{\circ} \mathrm{C}\right)$ for compounds $9 \mathrm{f}$ and $9 \mathrm{~g}$

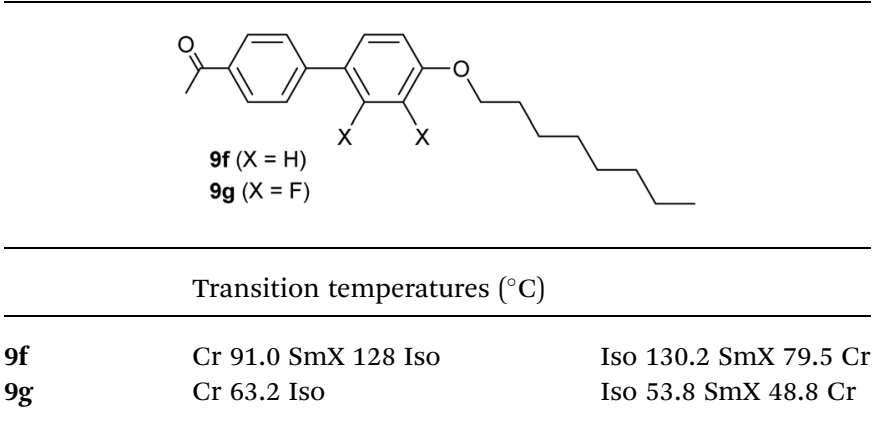

Table 3 Enthalpies $\left(\mathrm{kJ} \mathrm{mol}^{-1}\right)$ and dimensionless entropies associated with transitions for compounds $9 f$ and $9 g$

\begin{tabular}{llllllc}
\hline & & Cr-Iso & Cr-SmX & SmX-Iso & Iso-SmX & SmX-Cr \\
\hline 9f & $\Delta H$ & - & 23.48 & 13.377 & 12.54 & 24.10 \\
& $\Delta S / R$ & - & 7.755 & 4.0106 & 3.739 & 8.819 \\
9g & $\Delta H$ & 25.91 & - & - & 2.713 & 22.95 \\
& $\Delta S / R$ & 9.265 & - & - & 0.9980 & 8.574
\end{tabular}

to demonstrate liquid crystalline properties. Neither $\mathbf{3 f}$ nor $\mathbf{3 g}$ exhibited a mesophase in the first heating cycle, but upon cooling, a nematic phase appeared (Fig. 4). Further cooling did not result in crystallization, instead a glass transition occurred and when reheating the glass, the nematic phase reappeared and the transition from nematic to isotropic liquid could also
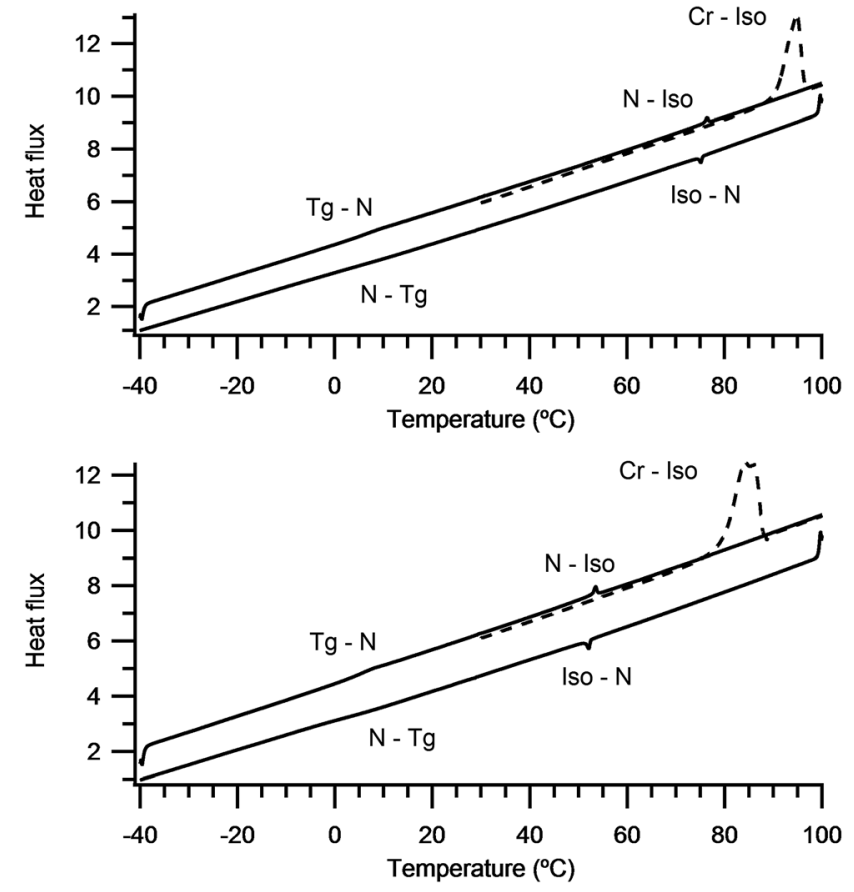

Fig. 4 DSC thermograms at a heating-cooling rate of $5^{\circ} \mathrm{C} \mathrm{min}^{-1}$ for compound $3 \mathrm{f}$ (Top) and compound $3 \mathrm{~g}$ (Bottom). The dashed line corresponds to the first heating. 
be observed. DHA $3 f$ gave a melt at $90.6{ }^{\circ} \mathrm{C}$ and the nematic phase appeared upon cooling at $75.7^{\circ} \mathrm{C}$, see Fig. 4 . On heating, the clearing point was found to be at $75.6{ }^{\circ} \mathrm{C}$. The phase transitions showed small enthalpy changes, which agrees with the changes from nematic to liquid phase and vice versa. For the fluorinated $3 \mathrm{~g}$ analogue, the melt occurred at a temperature about $10{ }^{\circ} \mathrm{C}$ lower than for $3 f$. The clearing point for the nematic phase of $3 \mathrm{~g}$ is $52.6{ }^{\circ} \mathrm{C}$, while it is $75.7{ }^{\circ} \mathrm{C}$ for $3 \mathrm{f}$. Table 4 lists transition temperatures for $\mathbf{3 f}$ and $\mathbf{3 g}$ and Table 5 the enthalpies and entropies associated with the transitions.

When 3f is irradiated (at $365 \mathrm{~nm}$ ) in the nematic phase, a color change from yellow-orange to red can be observed by the naked eye, but this did not correspond to a change in the phases. When solid VHF 17 was heated to $40{ }^{\circ} \mathrm{C}$ for $24 \mathrm{~h}$, neither a change in color nor a phase change was observed, while heating the same material to $80{ }^{\circ} \mathrm{C}$ resulted in a simultaneous melt and color change to yellow ascribed to conversion to the DHA state. When $\mathbf{3 g}$ was irradiated with light in the nematic phase at $40{ }^{\circ} \mathrm{C}$ for $60 \mathrm{~min}$, conversion to the corresponding VHF occurred in a ratio of $3: 7(\mathrm{VHF} / \mathrm{DHA})$ as determined by NMR spectroscopy. After one hour of irradiation, using POM one could see an increase in the alignment of the nematic phase, such that the defects disappear (Fig. 5c). Irradiation for a prolonged period (16 hours) afforded no further changes in neither the POM picture nor in the isomer ratio determined by NMR spectroscopy. When left in the dark to reclose to form the DHA, the alignment is maintained. If the top glass slide is sheared horizontally to disrupt the alignment, the nematic texture appears for the DHA, (Fig. 5d). If the same disruption is done with the VHF present, the alignment reforms over several minutes. In addition to the VHF structure being planar, it also

Table 4 Transition temperatures $\left({ }^{\circ} \mathrm{C}\right)$ for compounds $3 \mathrm{f}$ and $3 \mathrm{~g}$

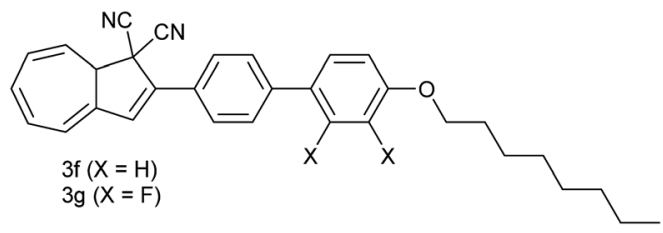

Transition temperatures $\left({ }^{\circ} \mathrm{C}\right)$

$3 f$

$3 g$

Table 5 The enthalpies $\left(\mathrm{kJ} \mathrm{mol}^{-1}\right.$ ) and dimensionless entropies associated with transition for compound $3 \mathrm{f}$ and $3 \mathrm{~g}$

\begin{tabular}{lllll}
\hline & & N-Iso & Cr-Iso & Iso-N \\
\hline 3f & $\Delta H$ & 0.4612 & 30.53 & 0.4044 \\
& $\Delta S / R$ & 0.1590 & 10.09 & 0.1394 \\
$3 \mathrm{~g}$ & $\Delta H$ & 0.4526 & 31.70 & 0.4057 \\
& $\Delta S / R$ & 0.1671 & 10.80 & 0.1498
\end{tabular}

has a larger dipole moment, which could in essence lead to this larger degree of alignment of the total system relative to the corresponding neat nematic DHA. There are several examples in the literature ${ }^{22}$ with photoalignment; the difference is that our experiment was performed in between untreated glass slides, which gives a texture. This texture then disappears upon irradiation. On the other hand, when heating pure VHF 17, no mesophases were observed for VHF 17, instead a rapid ringclosure to $3 \mathbf{f}$ occurred.

\section{Conclusions}

In order to ascertain the requirements of substitution needed to make the DHA photoswitch liquid crystalline, we have prepared a large selection of compounds with various substituents at position 2 of the DHA core. Of these compounds, two exhibited a nematic liquid crystal phase, both of which had an octyloxybiaryl attached to $\mathrm{C} 2$ of DHA. Irradiation of the one compound, with fluorine substitution of the biaryl, in its nematic phase partly formed the VHF. This DHA/VHF mixture exhibited a higher degree of alignment. In time, the DHA was regenerated and the order maintained. Nevertheless, full conversion to the VHF from the DHA in the nematic phase was not possible. This work presents an important step in using the DHA-VHF system in photoactive liquid crystalline materials. Future work will be aiming at exploring the influence of having two substituent groups on the DHA core.

\section{Experimental}

\subsection{General methods}

Chemicals were used as purchased from commercial sources. Purification of products was carried out by flash chromatography on silica gel $(40-63 \mu \mathrm{m}, 60 \AA)$. Thin-layer chromatography (TLC) was carried out using aluminum sheets precoated with silica gel. ${ }^{1} \mathrm{H}$ NMR (500 $\left.\mathrm{MHz}\right)$ and ${ }^{13} \mathrm{C}$ NMR $(125 \mathrm{MHz})$ spectra were recorded on an instrument with a noninverse cryoprobe using the residual solvent as the internal standard $\left(\mathrm{CDCl}_{3},{ }^{1} \mathrm{H} 7.26 \mathrm{ppm}\right.$ and $\left.{ }^{13} \mathrm{C} 77.16 \mathrm{ppm}\right)$. All chemical shifts are quoted on the $\delta$ scale (ppm), and all coupling constants $(J)$ are expressed in $\mathrm{Hz}$. In APT spectra, $\mathrm{CH}$ and $\mathrm{CH}_{3}$ correspond to negative signals and $\mathrm{C}$ and $\mathrm{CH}_{2}$ correspond to positive signals. High resolution mass spectra (HRMS) were acquired either using an electrospray method of ionization (ESP) or using MALDI. Melting points are uncorrected. Compounds 15 (ref. 23) and 16 (ref. 24) were made by their respective literature methods.

General procedure for 7a-e. A mixture consisting of 4-cyanophenol 6, the dibromide 5 (either a-e; 2-2.5 molar equivalents) and $\mathrm{K}_{2} \mathrm{CO}_{3}$ (1.5-2 molar equivalents) in acetone $(200 \mathrm{~mL})$ was heated to reflux point for $16 \mathrm{~h}$. The contents of the vessel were allowed to cool to rt and filtered. The solvent was removed from the filtrate and the crude residue was subjected to column chromatography (gradient elution of petroleum spirit to toluene) to afford 7 (either a-e) as a white solid.

4-((5-Bromopentyl)oxy)benzonitrile (7a). $\mathrm{Mp}=54.0-55.9{ }^{\circ} \mathrm{C} .{ }^{1} \mathrm{H}$ $\operatorname{NMR}\left(500 \mathrm{MHz}, \mathrm{CDCl}_{3}\right): \delta=7.57(\mathrm{~d}, J=8.8 \mathrm{~Hz}, 2 \mathrm{H}), 6.93(\mathrm{~d}, J=$ 


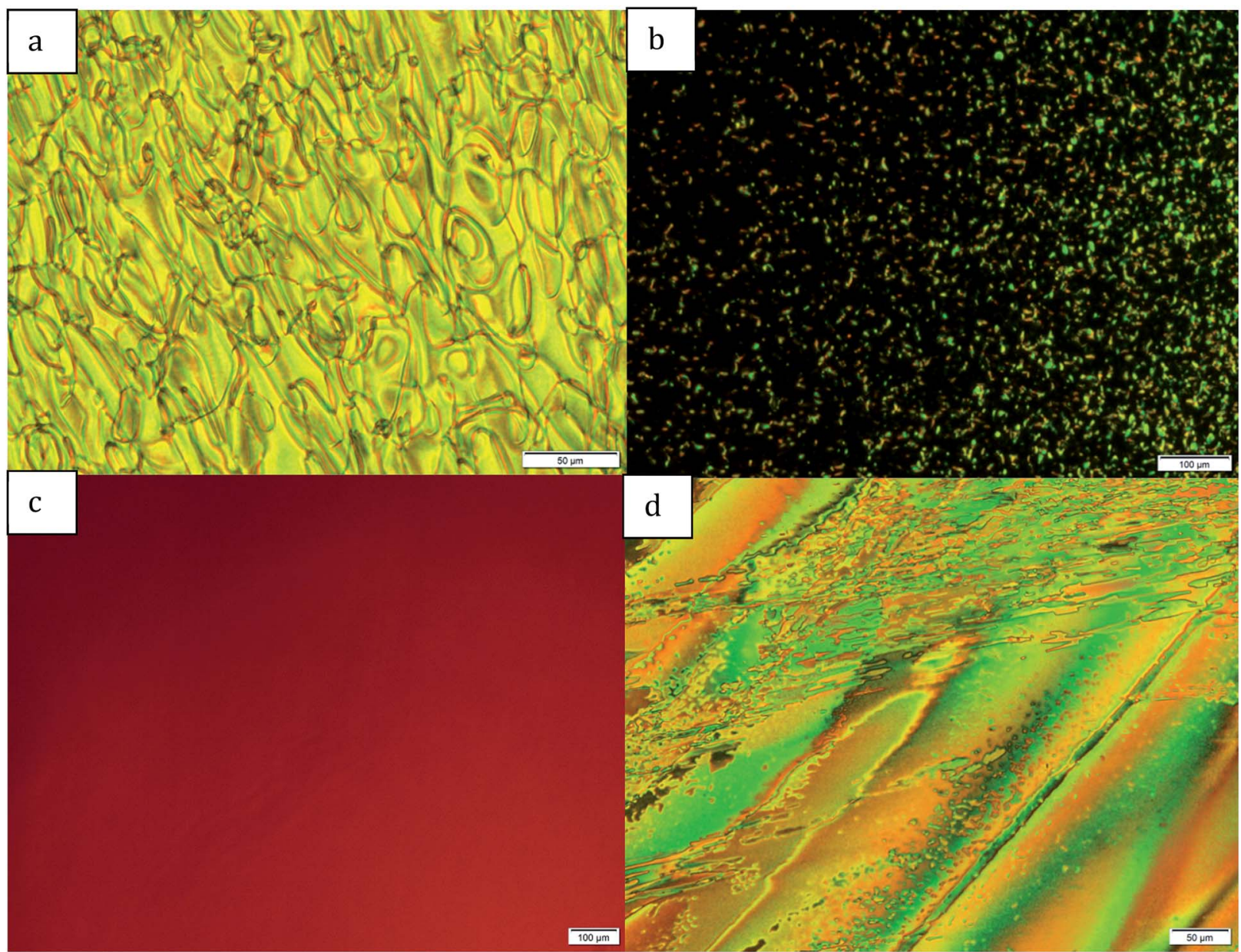

Fig. 5 (a) Photomicrographs of the nematic phase of $3 f$ at $60^{\circ} \mathrm{C}$. (b-d) Photomicrographs of the nematic phase of $3 g$ at $40{ }^{\circ} \mathrm{C}$, (b) before light, (c) after 60 min of light, (d) after dark for $16 \mathrm{~h}$

$8.8 \mathrm{~Hz}, 2 \mathrm{H}), 4.01(\mathrm{t}, J=6.3 \mathrm{~Hz}, 2 \mathrm{H}), 3.44(\mathrm{t}, J=6.7 \mathrm{~Hz}, 2 \mathrm{H}), 1.97-$ $1.91(\mathrm{~m}, 2 \mathrm{H}), 1.87-1.81(\mathrm{~m}, 2 \mathrm{H}), 1.67-1.60(\mathrm{~m}, 2 \mathrm{H}) \mathrm{ppm} .{ }^{13} \mathrm{C}$ NMR (125 MHz, $\left.\mathrm{CDCl}_{3}\right): \delta=162.4,134.1,119.4,115.3,104.0$, 68.1, 33.6, 32.5, 28.3, $24.8 \mathrm{ppm}$. MS (ESP +ve): $\mathrm{m} / z=290[(\mathrm{M}+$ $\left.\mathrm{Na})^{+}\right]$. Analysis calcd (\%) for $\mathrm{C}_{12} \mathrm{H}_{14} \mathrm{BrNO}$ (268.15): C 53.75, H 5.26, N 5.22; found: C 53.75, H 4.90, N 5.13.

4-((6-Bromohexyl)oxy)benzonitrile (7b). Mp $=44.0-46.5{ }^{\circ} \mathrm{C} .{ }^{1} \mathrm{H}$ NMR (500 MHz, $\left.\mathrm{CDCl}_{3}\right): \delta=7.57(\mathrm{~d}, J=8.9 \mathrm{~Hz}, 2 \mathrm{H}), 6.93(\mathrm{~d}, J=$ $8.9 \mathrm{~Hz}, 2 \mathrm{H}), 4.00(\mathrm{t}, J=6.4 \mathrm{~Hz}, 2 \mathrm{H}), 3.43(\mathrm{t}, J=6.6 \mathrm{~Hz}, 2 \mathrm{H}), 1.90$ $(\mathrm{p}, J=6.6 \mathrm{~Hz}, 2 \mathrm{H}), 1.82(\mathrm{p}, J=6.6 \mathrm{~Hz}, 2 \mathrm{H}), 1.53-1.50(\mathrm{~m}, 4 \mathrm{H})$ ppm. ${ }^{13} \mathrm{C}$ NMR $\left(125 \mathrm{MHz}, \mathrm{CDCl}_{3}\right): \delta=162.5,134.1,119.4,115.3$, 103.9, 68.3 33.8, 32.7, 29.0, 28.0, $25.3 \mathrm{ppm}$. MS (ESP +ve): $\mathrm{m} / z=$ $282\left[(\mathrm{M}+\mathrm{H})^{+}\right]$. Analysis calcd (\%) for $\mathrm{C}_{13} \mathrm{H}_{16} \mathrm{BrNO}$ (282.18): C 55.33, H 5.72, N 4.96; found: C 55.66, H 5.72, N 4.98.

4-((8-Bromooctyl)oxy)benzonitrile (7c). $R_{\mathrm{f}}=0.50$ (toluene). Mp $=68.5-69.8{ }^{\circ} \mathrm{C} .{ }^{1} \mathrm{H}$ NMR $\left(500 \mathrm{MHz}, \mathrm{CDCl}_{3}\right): \delta=7.57(\mathrm{~d}, J=8.8$ $\mathrm{Hz}, 2 \mathrm{H}), 6.93(\mathrm{~d}, J=8.8 \mathrm{~Hz}, 2 \mathrm{H}), 3.99(\mathrm{t}, J=6.5 \mathrm{~Hz}, 2 \mathrm{H}), 3.41(\mathrm{t}, J$ $=6.8 \mathrm{~Hz}, 2 \mathrm{H}), 1.89-1.84(\mathrm{~m}, 2 \mathrm{H}), 1.82-1.77(\mathrm{~m}, 2 \mathrm{H}), 1.49-1.42$ $(\mathrm{m}, 4 \mathrm{H}), 1.39-1.33(\mathrm{~m}, 4 \mathrm{H}) \mathrm{ppm} .{ }^{13} \mathrm{C} \mathrm{NMR}\left(125 \mathrm{MHz}, \mathrm{CDCl}_{3}\right): \delta=$ 162.5, 134.1, 119.4, 115.3, 103.8, 68.5, 34.1, 32.9, 29.2 , 29.1, 28.8, 28.2, 26.0 ppm. MS (ESP +ve): $m / z=310\left[(\mathrm{M}+\mathrm{H})^{+}\right]$. Analysis calcd (\%) for $\mathrm{C}_{15} \mathrm{H}_{20}$ BrNO (310.24): C 58.07, $\mathrm{H}$ 6.50, N 4.51; found: C 58.26, H 6.53, N 4.51.

4-((9-Bromononyl)oxy)benzonitrile (7d). $R_{\mathrm{f}}=0.50$ (toluene). Mp $=64.1-65.7{ }^{\circ} \mathrm{C} .{ }^{1} \mathrm{H}$ NMR $\left(500 \mathrm{MHz}, \mathrm{CDCl}_{3}\right): \delta=7.55(\mathrm{~d}, J=8.9$ $\mathrm{Hz}, 2 \mathrm{H}), 6.91(\mathrm{~d}, J=8.9 \mathrm{~Hz}, 2 \mathrm{H}), 3.98(\mathrm{t}, J=6.5 \mathrm{~Hz}, 2 \mathrm{H}), 3.39(\mathrm{t}, J$ $=6.5 \mathrm{~Hz}, 2 \mathrm{H}), 1.86-1.75(\mathrm{~m}, 4 \mathrm{H}), 1.46-1.39(\mathrm{~m}, 4 \mathrm{H}), 1.36-1.29$ $(\mathrm{m}, 6 \mathrm{H}) \mathrm{ppm} .{ }^{13} \mathrm{C}$ NMR (125 MHz, $\left.\mathrm{CDCl}_{3}\right): \delta=162.5,134.0$, 119.4, 115.2, 103.7, 68.4, 34.1, 32.8, 29.3, 29.2, 29.0, 28.7, 28.2, $25.9 \mathrm{ppm}$. MS (ESP +ve): $m / z=324\left[(\mathrm{M}+\mathrm{H})^{+}\right]$. Analysis calcd $(\%)$ for $\mathrm{C}_{16} \mathrm{H}_{22} \mathrm{BrNO}$ (324.26): $\mathrm{C} 59.27, \mathrm{H} 6.84, \mathrm{~N} \mathrm{4.32}$; found: $\mathrm{C}$ 59.52, H 6.93, N 4.32.

4-((10-Bromodecyl)oxy)benzonitrile (7e). $R_{\mathrm{f}}=0.50$ (toluene). $\mathrm{Mp}=73.2-74.7{ }^{\circ} \mathrm{C} .{ }^{1} \mathrm{H}$ NMR $\left(500 \mathrm{MHz}, \mathrm{CDCl}_{3}\right): \delta=7.57(\mathrm{~d}, J=$ $8.9 \mathrm{~Hz}, 2 \mathrm{H}), 6.93(\mathrm{~d}, J=8.9 \mathrm{~Hz}, 2 \mathrm{H}), 3.99(\mathrm{t}, J=6.5 \mathrm{~Hz}, 2 \mathrm{H}), 3.41$ $(\mathrm{t}, J=6.8 \mathrm{~Hz}, 2 \mathrm{H}), 1.88-1.77(\mathrm{~m}, 4 \mathrm{H}), 1.48-1.40(\mathrm{~m}, 4 \mathrm{H}), 1.36-$ $1.28(\mathrm{~m}, 8 \mathrm{H}) \mathrm{ppm} .{ }^{13} \mathrm{C} \mathrm{NMR}\left(125 \mathrm{MHz}, \mathrm{CDCl}_{3}\right): \delta=162.6,134.1$, 119.5, 115.3, 103.8, 68.5, 34.2, 32.9, 29.5, 29.5, 29.4, 29.1, 28.9, 28.3, 26.1 ppm. MS (ESP +ve): $m / z=360\left[(\mathrm{M}+\mathrm{Na})^{+}\right]$. Analysis calcd (\%) for $\mathrm{C}_{17} \mathrm{H}_{24} \mathrm{BrNO}$ (338.29): $\mathrm{C}$ 60.36, $\mathrm{H}$ 7.15, N 4.14; found: C 60.53, H 7.19, N 4.15.

General procedure for 9a-e. A mixture consisting of 4hydroxyacetophenone $\mathbf{8}$, bromide 7 (either a-e; 0.8-0.85 molar 
equivalents) and $\mathrm{K}_{2} \mathrm{CO}_{3}$ (1-2 molar equivalents) in acetone (100 $\mathrm{mL}$ ) was heated to reflux point for $24 \mathrm{~h}$. The contents of the vessel were allowed to cool to rt, diluted with $\mathrm{CH}_{2} \mathrm{Cl}_{2}(200 \mathrm{~mL})$ and filtered. The solvent was removed from the filtrate and the crude residue was passed through a short $\mathrm{SiO}_{2}$ column $\left(\mathrm{CH}_{2} \mathrm{Cl}_{2}\right)$ to afford 9 (either a-e) as a white solid.

4-((5-(4-Acetylphenoxy)pentyl)oxy)benzonitrile (9a). $R_{\mathrm{f}}=0.39$. $\mathrm{Mp}=100.8-102.8{ }^{\circ} \mathrm{C} .{ }^{1} \mathrm{H}$ NMR $\left(500 \mathrm{MHz}, \mathrm{CDCl}_{3}\right): \delta=7.96(\mathrm{~d}, J$ $=8.9 \mathrm{~Hz}, 2 \mathrm{H}), 7.60(\mathrm{~d}, J=8.9 \mathrm{~Hz}, 2 \mathrm{H}), 6.93(\mathrm{~d}, J=8.9 \mathrm{~Hz}, 2 \mathrm{H})$, $6.92(\mathrm{~d}, J=8.9 \mathrm{~Hz}, 2 \mathrm{H}), 4.06(\mathrm{t}, J=6.2 \mathrm{~Hz}, 2 \mathrm{H}), 4.04(\mathrm{t}, J=6.3 \mathrm{~Hz}$, $2 \mathrm{H}), 2.55$ (s, 3H), 1.92-1.87 (m, 4H), 1.70-1.64 (m, 2H) ppm. ${ }^{13} \mathrm{C}$ NMR (125 MHz, $\left.\mathrm{CDCl}_{3}\right): \delta=196.9,163.0,162.4,134.1,130.7$, 130.4, 119.4, 115.3, 114.2, 104.0, 68.2 , 68.0, 29.0, 28.9, 26.5, 22.8 ppm. HRMS (MALDI +ve) calcd for $\mathrm{C}_{20} \mathrm{H}_{22} \mathrm{NO}_{3}\left([\mathrm{M}+\mathrm{H}]^{+}\right): m / z=$ 324.1594; exp 324.1595. Analysis calcd (\%) for $\mathrm{C}_{20} \mathrm{H}_{21} \mathrm{NO}_{3}$ (323.39): C 74.28, H 6.55, N 4.33; found: C 74.09, H 6.28, N 4.27.

4-((6-(4-Acetylphenoxy)hexyl)oxy)benzonitrile (9b). $R_{\mathrm{f}}=0.33$. $\mathrm{Mp}=99.5-101.3{ }^{\circ} \mathrm{C} .{ }^{1} \mathrm{H} \mathrm{NMR}\left(500 \mathrm{MHz}, \mathrm{CDCl}_{3}\right): \delta=7.92(\mathrm{~d}, J=$ $8.9 \mathrm{~Hz}, 2 \mathrm{H}), 7.57(\mathrm{~d}, J=8.9 \mathrm{~Hz}, 2 \mathrm{H}), 6.93(\mathrm{~d}, J=8.9 \mathrm{~Hz}, 2 \mathrm{H}), 6.91$ $(\mathrm{d}, J=8.9 \mathrm{~Hz}, 2 \mathrm{H}), 4.04(\mathrm{t}, J=6.4 \mathrm{~Hz}, 2 \mathrm{H}), 4.02(\mathrm{t}, J=6.4 \mathrm{~Hz}$, $2 \mathrm{H}), 2.55$ (s, 3H), $1.87-1.82(\mathrm{~m}, 4 \mathrm{H}), 1.57-1.55(\mathrm{~m}, 4 \mathrm{H}) \mathrm{ppm} .{ }^{13} \mathrm{C}$ NMR (125 MHz, $\mathrm{CDCl}_{3}$ ): $\delta=196.9,163.1,162.5,134.1,130.7$, 130.4, 119.4, 115.3, 114.2, 103.9, 68.3, 68.1, 29.2, 29.1, 26.5, 25.9, 25.9 ppm. HRMS (MALDI +ve) calcd for $\mathrm{C}_{21} \mathrm{H}_{24} \mathrm{NO}_{3}\left([\mathrm{M}+\mathrm{H}]^{+}\right)$: $m / z=338.1751$; $\exp 338.1751$. Analysis calcd (\%) for $\mathrm{C}_{21} \mathrm{H}_{23} \mathrm{NO}_{3}$ (337.42): C 74.75, H 6.87, N 4.15; found: C 74.60, H 6.73, N 3.99.

4-((8-(4-Acetylphenoxy)octyl)oxy)benzonitrile (9c). $R_{\mathrm{f}}=0.35 . \mathrm{Mp}$ $=91.1-92.3{ }^{\circ} \mathrm{C} .{ }^{1} \mathrm{H}$ NMR $\left(500 \mathrm{MHz}, \mathrm{CDCl}_{3}\right): \delta=7.92(\mathrm{~d}, J=8.9$ $\mathrm{Hz}, 2 \mathrm{H}), 7.56$ (d, $J=8.8 \mathrm{~Hz}, 2 \mathrm{H}), 6.92(\mathrm{~d}, J=8.9 \mathrm{~Hz}, 2 \mathrm{H}), 6.91$ (d, $J=8.8 \mathrm{~Hz}, 2 \mathrm{H}), 4.02(\mathrm{t}, J=6.5 \mathrm{~Hz}, 2 \mathrm{H}), 3.99(\mathrm{~d}, J=6.5 \mathrm{~Hz}, 2 \mathrm{H})$, $2.55(\mathrm{~s}, 3 \mathrm{H}), 1.84-1.78(\mathrm{~m}, 4 \mathrm{H}), 1.50-1.45(\mathrm{~m}, 4 \mathrm{H}), 1.41-1.38(\mathrm{~m}$, $4 \mathrm{H}) \mathrm{ppm} .{ }^{13} \mathrm{C} \mathrm{NMR}\left(125 \mathrm{MHz}, \mathrm{CDCl}_{3}\right): \delta=196.9,163.2,162.5$, $134.1,130.7,130.3,119.4,115.3,114.2$, 103.8, 68.5, 68.3, 29.4, 29.4, 29.2, 29.1, 26.5, 26.1, 26.0 ppm. HRMS (MALDI +ve) calcd for $\mathrm{C}_{23} \mathrm{H}_{28} \mathrm{NO}_{3} \mathrm{Na}\left([\mathrm{M}+\mathrm{Na}]^{+}\right): m / z=388.1883$; $\exp 388.1884$. Analysis calcd (\%) for $\mathrm{C}_{23} \mathrm{H}_{27} \mathrm{NO}_{3}$ (365.47): $\mathrm{C} 75.59, \mathrm{H} 7.45, \mathrm{~N}$ 3.83; found: C 75.51, H 7.28, N 3.78.

4-((9-(4-Acetylphenoxy)nonyl)oxy)benzonitrile (9d). $R_{\mathrm{f}}=0.35$. $\mathrm{Mp}=86.4-87.2{ }^{\circ} \mathrm{C} .{ }^{1} \mathrm{H}$ NMR $\left(500 \mathrm{MHz}, \mathrm{CDCl}_{3}\right): \delta=7.92(\mathrm{~d}, J=$ $8.6 \mathrm{~Hz}, 2 \mathrm{H}), 7.57$ (d, $J=8.6 \mathrm{~Hz}, 2 \mathrm{H}), 6.93(\mathrm{~d}, J=8.6 \mathrm{~Hz}, 2 \mathrm{H}), 6.91$ $(\mathrm{d}, J=8.6 \mathrm{~Hz}, 2 \mathrm{H}), 4.02(\mathrm{t}, J=6.5 \mathrm{~Hz}, 2 \mathrm{H}), 3.99(\mathrm{t}, J=6.5 \mathrm{~Hz}$, 2H), 2.55 (s, 3H), 1.83-1.77 (m, 4H), 1.49-1.43 (m, 4H), 1.39-1.35 $(\mathrm{m}, 6 \mathrm{H}) \mathrm{ppm} .{ }^{13} \mathrm{C} \mathrm{NMR}\left(125 \mathrm{MHz}, \mathrm{CDCl}_{3}\right): \delta=196.9,163.2$, 162.6, 134.1, 130.7, 130.3, 119.5, 115.3, 114.3, 103.8, 68.5, 68.3, 29.6, 29.4, 29.4 29.2, 29.1, 26.5, 26.1, 26.1 ppm. HRMS (MALDI +ve) calcd for $\mathrm{C}_{24} \mathrm{H}_{30} \mathrm{~N}_{3} \mathrm{O}_{2}\left([\mathrm{M}+\mathrm{H}]^{+}\right): m / z=380.2220$; exp 380.2221. Analysis calcd (\%) for $\mathrm{C}_{24} \mathrm{H}_{29} \mathrm{NO}_{3}$ (379.50): $\mathrm{C} 75.96, \mathrm{H}$ 7.70, N 3.69; found: C 75.82, H 7.68, N 3.55.

4-((10-(4-Acetylphenoxy)decyl)oxy)benzonitrile (9e). $R_{\mathrm{f}}=0.40$. $\mathrm{Mp}=71.5-73.5{ }^{\circ} \mathrm{C} .{ }^{1} \mathrm{H}$ NMR $\left(500 \mathrm{MHz}, \mathrm{CDCl}_{3}\right): \delta=7.92(\mathrm{~d}, J=$ $8.8 \mathrm{~Hz}, 2 \mathrm{H}), 7.56(\mathrm{~d}, J=8.8 \mathrm{~Hz}, 2 \mathrm{H}), 6.92(\mathrm{~d}, J=8.8 \mathrm{~Hz}, 2 \mathrm{H}), 6.91$ $(\mathrm{d}, J=8.8 \mathrm{~Hz}, 2 \mathrm{H}), 4.01(\mathrm{t}, J=6.5 \mathrm{~Hz}, 2 \mathrm{H}), 3.99(\mathrm{~d}, J=6.5 \mathrm{~Hz}$, 2H), 2.55 (s, 3H), 1.83-1.76 (m, 4H), 1.48-1.42 (m, 4H), 1.37-1.32 $(\mathrm{m}, 8 \mathrm{H}) \mathrm{ppm} .{ }^{13} \mathrm{C} \mathrm{NMR}\left(125 \mathrm{MHz}, \mathrm{CDCl}_{3}\right): \delta=196.9,163.2$, 162.6, 134.1, 130.7, 130.3, 119.4, 115.3, 114.2, 103.8, 68.5, 68.4, 29.6, 29.4, 29.4, 29.2, 29.1, 26.5, 26.1, 26.1 ppm, 1C masked. HRMS (MALDI +ve) calcd for $\mathrm{C}_{25} \mathrm{H}_{32} \mathrm{NO}_{3}\left([\mathrm{M}+\mathrm{H}]^{+}\right): m / z=$
394.2377; exp 394.2377. Analysis calcd (\%) for $\mathrm{C}_{25} \mathrm{H}_{31} \mathrm{NO}_{3}$ (393.53): C 76.30, H 7.94, N 3.56; found: C 76.29, H 7.90, N 3.52. 1-(4'-(Octyloxy)-[1,1'-biphenyl]-4-yl)ethan-1-one $\quad(\mathbf{9 f}) .^{\mathbf{2 0 , 2 1}} \quad$ To a degassed biphasic mixture of 4-iodoacetophenone 10 (5.03 g, $20.4 \mathrm{mmol}), \mathrm{K}_{3} \mathrm{PO}_{4}(11.94 \mathrm{~g}, 56.71 \mathrm{mmol})$ and $11 \mathrm{f}(6.43 \mathrm{~g}, 25.7$ $\mathrm{mmol})$ in toluene $(120 \mathrm{~mL})$ and water $(20 \mathrm{~mL})$ was added $\mathrm{Pd}\left(\mathrm{PPh}_{3}\right)_{4}(1.00 \mathrm{~g}, 0.86 \mathrm{mmol})$ and the biphasic mixture was stirred $20 \mathrm{~h}$ at $\mathrm{rt}$ and heated to $80{ }^{\circ} \mathrm{C}$ for $10 \mathrm{~h}$. The reaction mixture was cooled to rt and was diluted with water $(80 \mathrm{~mL})$ and the phases were separated. The aqueous phase was extracted with $\mathrm{CH}_{2} \mathrm{Cl}_{2}(2 \times 50 \mathrm{~mL})$ and the combined organic extracts dried over $\mathrm{MgSO}_{4}$, filtered and the solvent removed in vacuo. The solid was triturated with $\mathrm{CH}_{2} \mathrm{Cl}_{2}$ and heptane to give $9 f$ $(5.73 \mathrm{~g}, 86 \%)$ as an off white solid. $R_{\mathrm{f}}=0.41$ (toluene). $\mathrm{Mp}=\mathrm{Cr}$ 91.0 SmX 128 Iso; Iso $130.2 \mathrm{SmX} 79.5 \mathrm{Cr}{ }^{\circ} \mathrm{C} .{ }^{1} \mathrm{H}$ NMR $(500 \mathrm{MHz}$, $\left.\mathrm{CDCl}_{3}\right): \delta=8.00(\mathrm{~d}, J=8.4 \mathrm{~Hz}, 2 \mathrm{H}), 7.64(\mathrm{~d}, J=8.4 \mathrm{~Hz}, 2 \mathrm{H}), 7.57$ $(\mathrm{d}, J=8.6 \mathrm{~Hz}, 2 \mathrm{H}), 6.99(\mathrm{~d}, J=8.6 \mathrm{~Hz}, 2 \mathrm{H}), 4.01(\mathrm{t}, J=6.5 \mathrm{~Hz}$, $2 \mathrm{H}), 2.63(\mathrm{~s}, 3 \mathrm{H}), 1.84-1.78(\mathrm{~m}, 2 \mathrm{H}), 1.50-1.45$ (m, 2H), 1.42-1.20 $(\mathrm{m}, 8 \mathrm{H}), 0.89(\mathrm{t}, J=6.8 \mathrm{~Hz}, 3 \mathrm{H}) \mathrm{ppm} .{ }^{13} \mathrm{C}$ NMR $(125 \mathrm{MHz}$, $\left.\mathrm{CDCl}_{3}\right): \delta=197.9,159.7,145.6,135.4,132.1,129.1,128.5,126.7$, $115.1,68.3,32.0,29.5,29.4,26.8,26.2,22.8,14.3 \mathrm{ppm}, 1 \mathrm{C}$ masked. HRMS (MALDI +ve) calcd for $\mathrm{C}_{22} \mathrm{H}_{28} \mathrm{O}_{2} \mathrm{Na}\left[(\mathrm{M}+\mathrm{Na})^{+}\right]$: $m / z=347.1982$; exp 347.1991. Analysis calcd (\%) for $\mathrm{C}_{22} \mathrm{H}_{28} \mathrm{O}_{2}$ (324.46): C 81.44, H 8.70; found: C 80.99, H 8.42.

1-(2',3'-Difluoro-4'-(octyloxy)-[1,1'-biphenyl]-4-yl)ethan-1-one (9g). To a degassed biphase of 4-iodoacetophenone 10 (5.20 g, 21.1 $\mathrm{mmol})$ in toluene $(120 \mathrm{~mL})$ and water $(20 \mathrm{~mL})$ were added $\mathrm{Pd}\left(\mathrm{PPh}_{3}\right)_{4}(1.00 \mathrm{~g}, 0.86 \mathrm{mmol}), \mathrm{K}_{3} \mathrm{PO}_{4}(14.90 \mathrm{~g}, 70.2 \mathrm{mmol})$ and $11 \mathrm{~g}$ ( $9.08 \mathrm{~g}, 31.7 \mathrm{mmol}$ ) and the biphasic mixture was stirred $20 \mathrm{~h}$ at $\mathrm{rt}$, followed by heating at $80^{\circ} \mathrm{C}$ for $7 \mathrm{~h}$. The reaction was allowed to cool to $\mathrm{rt}$ and the vessel was diluted with water $(80 \mathrm{~mL})$ and the phases were separated. The aqueous phase was extracted with $\mathrm{CH}_{2} \mathrm{Cl}_{2}(2 \times 50 \mathrm{~mL})$ and the combined organic phases dried over $\mathrm{MgSO}_{4}$, filtered and the solvent removed in vacuo. The residue was subjected to flash column chromatography (gradient elution of $50-80 \% \mathrm{CH}_{2} \mathrm{Cl}_{2} /$ heptane) and triturated from $\mathrm{CH}_{2} \mathrm{Cl}_{2}$ and heptane to give $9 \mathrm{~g}(5.01 \mathrm{~g}, 66 \%)$ as a white solid. $R_{\mathrm{f}}=0.51$ (toluene). $\mathrm{Mp}=\mathrm{Cr} 63.2$ Iso; Iso $53.8 \mathrm{SmX}$ $48.8 \mathrm{Cr}{ }^{\circ} \mathrm{C} .{ }^{1} \mathrm{H}$ NMR $\left(500 \mathrm{MHz}, \mathrm{CDCl}_{3}\right): \delta=8.02(\mathrm{~d}, J=7.9 \mathrm{~Hz}$, $2 \mathrm{H}), 7.61(\mathrm{~d}, J=7.9 \mathrm{~Hz}, 2 \mathrm{H}), 7.13(\mathrm{td}, J=8.4,2.2 \mathrm{~Hz}, 1 \mathrm{H}), 6.82$ (td, $J=8.4,1.6 \mathrm{~Hz}, 1 \mathrm{H}), 4.09(\mathrm{t}, J=6.5 \mathrm{~Hz}, 2 \mathrm{H}), 2.64(\mathrm{~s}, 3 \mathrm{H})$, 1.87-1.82 (m, 2H), 1.51-1.45 (m, 2H), 1.43-1.22 (m, 8H), $0.89(\mathrm{t}, J$ $=6.9 \mathrm{~Hz}, 3 \mathrm{H}) \mathrm{ppm} .{ }^{13} \mathrm{C} \mathrm{NMR}\left(125 \mathrm{MHz}, \mathrm{CDCl}_{3}\right): \delta=197.8,149.1$ (dd, $J=249.8,11.22 \mathrm{~Hz}$ ), 148.8 (dd, $J=8.3,3.1 \mathrm{~Hz}), 141.9$ (dd, $J$ $=248.2,14.9 \mathrm{~Hz}), 139.8(\mathrm{dd}, J=2.8,1.9 \mathrm{~Hz}), 136.2,129.0(\mathrm{~d}, J=$ $3.1 \mathrm{~Hz}), 128.7,123.8(\mathrm{t}, J=4.1 \mathrm{~Hz}), 121.8(\mathrm{~d}, J=10.7 \mathrm{~Hz}), 109.8$ (dd, $J=3.2,0.9 \mathrm{~Hz}$ ), 70.1, 32.0, 29.4, 29.4, 29.3, 26.8, 26.0, 22.8, 14.2 ppm. HRMS (MALDI +ve) calcd for $\mathrm{C}_{22} \mathrm{H}_{26} \mathrm{~F}_{2} \mathrm{O}_{2} \mathrm{Na}[(\mathrm{M}+$ $\left.\mathrm{Na})^{+}\right]: m / z=383.1793$; $\exp 383.1805$. Analysis calcd (\%) for $\mathrm{C}_{22} \mathrm{H}_{26} \mathrm{~F}_{2} \mathrm{O}_{2}$ (360.44): C 73.31, $\mathrm{H} 7.27$; found: $\mathrm{C} 73.25, \mathrm{H}$ 7.34.

2,3-Difluoro-4-(octyloxy)acetophenone (9h). ${ }^{\mathbf{1 4}}$ To a degassed solution of $12(6.22 \mathrm{~g}, 25.7 \mathrm{mmol})$ and $\mathrm{FeCl}_{3}(5.32 \mathrm{~g}, 32.8 \mathrm{mmol})$ in $\mathrm{CH}_{2} \mathrm{Cl}_{2}(150 \mathrm{~mL})$, under argon and in an ice bath, was added a solution of acetyl chloride $\left(2.1 \mathrm{~mL}\right.$ in $50 \mathrm{~mL} \mathrm{CH}_{2} \mathrm{Cl}_{2}, 29.4$ $\mathrm{mmol}$ ) dropwise over a period of $80 \mathrm{~min}$. The resulting mixture was stirred for $22 \mathrm{~h}$ during which time the vessel was allowed to reach $\mathrm{rt}$. The reaction was quenched with water $(500 \mathrm{~mL})$ and 
the phases separated. The aqueous phase was extracted with $\mathrm{CH}_{2} \mathrm{Cl}_{2}(100 \mathrm{~mL})$ and the combined organic extracts were washed with saturated aqueous saturated $\mathrm{NaHCO}_{3}(200 \mathrm{~mL})$, dried over $\mathrm{MgSO}_{4}$, filtered and concentrated in vacuo. The residue was purified by flash column chromatography (gradient elution of heptane to toluene) to give $9 \mathbf{h}(6.14 \mathrm{~g}, 84 \%)$ as a pale yellow oil. $R_{\mathrm{f}}=0.55$ (toluene). ${ }^{1} \mathrm{H}$ NMR (500 $\left.\mathrm{MHz}, \mathrm{CDCl}_{3}\right): \delta=$ 7.65 (ddd, $J=9.0,6.7,2.3 \mathrm{~Hz}, 1 \mathrm{H}), 6.78$ (ddd, $J=9.0,7.0,1.8 \mathrm{~Hz}$, $1 \mathrm{H}), 4.10(\mathrm{t}, J=6.6 \mathrm{~Hz}, 2 \mathrm{H}), 2.61$ (d, $J=5.0 \mathrm{~Hz}, 3 \mathrm{H}), 1.96-1.78$ $(\mathrm{m}, 2 \mathrm{H}), 1.52-1.41(\mathrm{~m}, 2 \mathrm{H}), 1.37-1.25(\mathrm{~m}, 8 \mathrm{H}), 0.88(\mathrm{t}, \mathrm{J}=6.8 \mathrm{~Hz}$, $3 \mathrm{H})$ ppm. ${ }^{13} \mathrm{C} \mathrm{NMR}\left(125 \mathrm{MHz}, \mathrm{CDCl}_{3}\right): \delta=193.9(\mathrm{dd}, J=3.8,2.4$ $\mathrm{Hz}), 152.8(\mathrm{dd}, J=8.1,3.8 \mathrm{~Hz}), 152.0(\mathrm{dd}, J=255.7,11.3 \mathrm{~Hz})$, $141.1(\mathrm{dd}, J=248.4,15.6 \mathrm{~Hz}), 125.0(\mathrm{dd}, J=4.1,3.4 \mathrm{~Hz}), 119.5$ (dd, $J=10.5,2.1), 108.9(\mathrm{dd}, J=2.7,1.0 \mathrm{~Hz}), 70.1,31.9,31.3(\mathrm{~d}, J$ $=7.2 \mathrm{~Hz}$ ), 29.4, 29.3, 29.1, 25.9, 22.8, 14.2 ppm. HRMS (ESP +ve) calcd for $\mathrm{C}_{16} \mathrm{H}_{22} \mathrm{~F}_{2} \mathrm{O}_{2} \mathrm{Na}\left[(\mathrm{M}+\mathrm{Na})^{+}\right]: m / z=307.1480$; exp 307.1483. Analysis calcd (\%) for $\mathrm{C}_{16} \mathrm{H}_{22} \mathrm{~F}_{2} \mathrm{O}_{2}$ (284.35): $\mathrm{C} 67.59, \mathrm{H}$ 7.80; found: C 67.69, H 7.71.

General procedure for 13a-i. A biphasic mixture of 9 (either a-i), malononitrile (3.5-9 molar equivalents), $\mathrm{NH}_{4} \mathrm{OAc}$ (3-10 molar equivalents) in toluene (100-300 mL) and $\mathrm{AcOH}(4-23$ $\mathrm{mL}$ ) was heated using a Dean-Stark apparatus for 3-10 h. The vessel was cooled, diluted with toluene and decanted into a separatory funnel and water was added. The phases were separated and the organic phase washed with water and brine. The organic phase was dried over $\mathrm{MgSO}_{4}$, filtered and the solvent removed in vacuo. For subsequent purification for obtaining 13 (either a-i), see ESI. $\dagger$

2-(1-(4-((5-(4-Cyanophenoxy)pentyl)oxy)phenyl)ethylidene)malononitrile (13a). Yellowish solid. $R_{\mathrm{f}}=0.65\left(\mathrm{CH}_{2} \mathrm{Cl}_{2}\right) . \mathrm{Mp}=83.2-$ 84.9, 95.6-100.3 ${ }^{\circ} \mathrm{C} .{ }^{1} \mathrm{H} \mathrm{NMR}\left(500 \mathrm{MHz}, \mathrm{CDCl}_{3}\right): \delta=7.60(\mathrm{~d}, J=$ $8.9 \mathrm{~Hz}, 2 \mathrm{H}), 7.57$ (d, $J=8.9 \mathrm{~Hz}, 2 \mathrm{H}) 6.97(\mathrm{~d}, J=8.9 \mathrm{~Hz}, 2 \mathrm{H}), 6.94$ $(\mathrm{d}, J=8.9 \mathrm{~Hz}, 2 \mathrm{H}), 4.06(\mathrm{t}, J=6.2 \mathrm{~Hz}, 2 \mathrm{H}), 4.04(\mathrm{t}, J=6.2 \mathrm{~Hz}, 2 \mathrm{H})$, 2.61 (s, 3H), 1.92-1.87 (m, 4H), 1.70-1.64 (m, 2H) ppm. ${ }^{13} \mathrm{C}$ NMR $\left(125 \mathrm{MHz}, \mathrm{CDCl}_{3}\right): \delta=174.1,162.7,162.4,134.1,130.0,127.9$, $119.4,115.3,115.0,113.8,113.5,104.0,82.0$, 68.2, 28.9, 28.8, 23.9, 22.8 ppm, 1C masked. HRMS (MALDI +ve) calcd for $\mathrm{C}_{23} \mathrm{H}_{22} \mathrm{~N}_{3} \mathrm{O}_{2}\left([\mathrm{M}+\mathrm{H}]^{+}\right): m / z=372.1707$; $\exp 372.1707$. Analysis calcd (\%) for $\mathrm{C}_{23} \mathrm{H}_{21} \mathrm{~N}_{3} \mathrm{O}_{2}$ (371.44): C 74.37, $\mathrm{H}$ 5.70, N 11.31; found: C 73.41, H 5.76, N 10.80.

2-(1-(4-((6-(4-Cyanophenoxy)hexyl)oxy)phenyl)ethylidene)malononitrile (13b). White solid. $R_{\mathrm{f}}=0.61\left(\mathrm{CH}_{2} \mathrm{Cl}_{2}\right) . \mathrm{Mp}=62.5-64.5$ ${ }^{\circ} \mathrm{C} .{ }^{1} \mathrm{H}$ NMR $\left(500 \mathrm{MHz}, \mathrm{CDCl}_{3}\right): \delta=7.60(\mathrm{~d}, J=8.9 \mathrm{~Hz}, 2 \mathrm{H}), 7.57$ $(\mathrm{d}, J=8.9 \mathrm{~Hz}, 2 \mathrm{H}) 6.97(\mathrm{~d}, J=8.9 \mathrm{~Hz}, 2 \mathrm{H}), 6.93(\mathrm{~d}, J=8.9 \mathrm{~Hz}$, $2 \mathrm{H}), 4.04(\mathrm{t}, J=6.4 \mathrm{~Hz}, 2 \mathrm{H}), 4.02(\mathrm{t}, J=6.3 \mathrm{~Hz}, 2 \mathrm{H}), 2.61(\mathrm{~s}, 3 \mathrm{H})$, 1.86-1.83 (m, 4H), 1.57-1.54 (m, 4H) ppm. ${ }^{13} \mathrm{C}$ NMR (125 MHz, $\left.\mathrm{CDCl}_{3}\right): \delta=174.1,162.8,162.5,134.1,130.0,127.9,119.4,115.3$, 115.0, 113.8, 113.5, 103.9, 82.0, 68.3, 29.1, 29.1, 25.9, 25.9, 23.9 ppm, 1C masked. HRMS (MALDI +ve) calcd for $\mathrm{C}_{24} \mathrm{H}_{24} \mathrm{~N}_{3} \mathrm{O}_{2}([\mathrm{M}$ $\left.+\mathrm{H}]^{+}\right): m / z=386.1858$; $\exp 386.1864$. Analysis calcd (\%) for $\mathrm{C}_{24} \mathrm{H}_{23} \mathrm{~N}_{3} \mathrm{O}_{2}$ (385.47): C 74.78, H 6.01, N 10.90; found: C 74.75, H 5.73, N 10.81 .

2-(1-(4-((8-(4-Cyanophenoxy)octyl)oxy)phenyl)ethylidene)malononitrile (13c). White solid. $R_{\mathrm{f}}=0.62\left(\mathrm{CH}_{2} \mathrm{Cl}_{2}\right) . \mathrm{Mp}=67.0-67.7^{\circ} \mathrm{C} .{ }^{1} \mathrm{H}$ $\operatorname{NMR}\left(500 \mathrm{MHz}, \mathrm{CDCl}_{3}\right): \delta=7.64(\mathrm{~d}, J=8.9 \mathrm{~Hz}, 2 \mathrm{H}), 7.60(\mathrm{~d}, J=8.9$ $\mathrm{Hz}, 2 \mathrm{H}) 7.00(\mathrm{~d}, J=8.9 \mathrm{~Hz}, 2 \mathrm{H}), 6.96(\mathrm{~d}, J=8.9 \mathrm{~Hz}, 2 \mathrm{H}), 4.05(\mathrm{t}, J=$ $6.5 \mathrm{~Hz}, 2 \mathrm{H}), 4.02(\mathrm{t}, J=6.4 \mathrm{~Hz}, 2 \mathrm{H}), 2.64(\mathrm{~s}, 3 \mathrm{H}), 1.87-1.81(\mathrm{~m}, 4 \mathrm{H})$,
1.54-1.48 (m, 4H), 1.46-1.41 ppm. ${ }^{13} \mathrm{C}$ NMR (125 MHz, $\left.\mathrm{CDCl}_{3}\right): \delta=$ 174.2, 162.8, 162.5, 134.1, 130.0, 127.7, 119.5, 115.2, 114.9, 113.9, 113.6, 103.7, 81.8, 68.4, 29.4, 29.1, 29.1, 26.0, 26.0, 24.0 ppm. HRMS (MALDI +ve) calcd for $\mathrm{C}_{26} \mathrm{H}_{28} \mathrm{~N}_{3} \mathrm{O}_{2}\left([\mathrm{M}+\mathrm{H}]^{+}\right): m / z=$ 414.2176; exp 414.2177. Analysis calcd (\%) for $\mathrm{C}_{26} \mathrm{H}_{27} \mathrm{~N}_{3} \mathrm{O}_{2}$ (413.52): C 75.52, H 6.58, N 10.16; found: C 75.13, H 6.29, N 10.19.

2-(1-(4-((9-(4-Cyanophenoxy)nonyl)oxy)phenyl)ethylidene)malononitrile (13d). White solid. $R_{\mathrm{f}}=0.68\left(\mathrm{CH}_{2} \mathrm{Cl}_{2}\right) . \mathrm{Mp}=87.1-88.1$ ${ }^{\circ} \mathrm{C} .{ }^{1} \mathrm{H}$ NMR $\left(500 \mathrm{MHz}, \mathrm{CDCl}_{3}\right): \delta=7.60(\mathrm{~d}, J=8.9 \mathrm{~Hz}, 2 \mathrm{H}), 7.57$ $(\mathrm{d}, J=8.8 \mathrm{~Hz}, 2 \mathrm{H}), 6.97(\mathrm{~d}, J=8.9 \mathrm{~Hz}, 2 \mathrm{H}), 6.93(\mathrm{~d}, J=8.8 \mathrm{~Hz}$, $2 \mathrm{H}), 4.01(\mathrm{t}, J=6.5 \mathrm{~Hz}, 2 \mathrm{H}), 3.99(\mathrm{t}, J=6.5 \mathrm{~Hz}, 2 \mathrm{H}), 2.61(\mathrm{~s}, 3 \mathrm{H})$, 1.83-1.77 (m, 4H), 1.49-1.43 (m, 4H), 1.39-1.35 (m, 6H) ppm. ${ }^{13} \mathrm{C}$ NMR (125 MHz, $\left.\mathrm{CDCl}_{3}\right): \delta=174.2,162.8,162.5,134.1$, 130.0, 127.7, 119.5, 115.3, 115.0, 113.9, 113.6, 103.7, 81.8, 68.5, 68.5, 29.6, 29.4, 29.1, 29.1, 26.1, 26.0, 23.9 ppm, 1C masked. HRMS (MALDI +ve) calcd for $\mathrm{C}_{27} \mathrm{H}_{29} \mathrm{~N}_{3} \mathrm{O}_{2} \mathrm{Na}\left([\mathrm{M}+\mathrm{Na}]^{+}\right): m / z=$ 450.2157; exp 450.2153. Analysis calcd (\%) for $\mathrm{C}_{27} \mathrm{H}_{29} \mathrm{~N}_{3} \mathrm{O}_{2}$ (427.55): C 75.85, H 6.84, N 9.83; found: C 75.50, H 6.81, N 9.80.

2-(1-(4-((10-(4-Cyanophenoxy)decyl)oxy)phenyl)ethylidene $)$ malononitrile (13e). White solid. $R_{\mathrm{f}}=0.65\left(\mathrm{CH}_{2} \mathrm{Cl}_{2}\right) . \mathrm{Mp}=62.6-63.8$ ${ }^{\circ} \mathrm{C} .{ }^{1} \mathrm{H}$ NMR $\left(500 \mathrm{MHz}, \mathrm{CDCl}_{3}\right): \delta=7.60(\mathrm{~d}, J=8.9 \mathrm{~Hz}, 2 \mathrm{H}), 7.57$ $(\mathrm{d}, J=8.8 \mathrm{~Hz}, 2 \mathrm{H}), 6.97(\mathrm{~d}, J=8.9 \mathrm{~Hz}, 2 \mathrm{H}), 6.93(\mathrm{~d}, J=8.8 \mathrm{~Hz}$, $2 \mathrm{H}), 4.01(\mathrm{t}, J=6.5 \mathrm{~Hz}, 2 \mathrm{H}), 3.99(\mathrm{t}, J=6.5 \mathrm{~Hz}, 2 \mathrm{H}), 2.61(\mathrm{~s}, 3 \mathrm{H})$, 1.83-1.77 (m, 4H), 1.47-1.43 (m, 4H), 1.37-1.31 (m, 8H) ppm. ${ }^{13} \mathrm{C}$ NMR (125 MHz, $\left.\mathrm{CDCl}_{3}\right): \delta=174.2,162.8,162.5,134.1,130.0$, $127.7,199.5$, 115.3, 115.0, 113.9, 113.6, 103.7, 81.8, 68.5, 68.5, 29.6, 29.4, 29.1, 29.1, 26.1, 26.0, 23.9 ppm, 2Cs masked. HRMS (MALDI +ve) calcd for $\mathrm{C}_{28} \mathrm{H}_{32} \mathrm{~N}_{3} \mathrm{O}_{2}\left([\mathrm{M}+\mathrm{H}]^{+}\right): m / z=442.2489$; exp 442.2491. Analysis calcd (\%) for $\mathrm{C}_{28} \mathrm{H}_{31} \mathrm{~N}_{3} \mathrm{O}_{2}$ (441.58): C, 76.16; H, 7.08; N, 9.52; found: C 76.30, H 7.07, N 9.50.

2-(1-(4'-(Octyloxy)-[1,1'-biphenyl]-4-yl)ethylidene)malononitrile (13f). White solid. $R_{\mathrm{f}}=0.56$ (toluene). $\mathrm{Mp}=58.6-59.4{ }^{\circ} \mathrm{C} .{ }^{1} \mathrm{H}$ NMR $\left(500 \mathrm{MHz}, \mathrm{CDCl}_{3}\right): \delta=7.68(\mathrm{~d}, J=8.5 \mathrm{~Hz}, 2 \mathrm{H}), 7.64(\mathrm{~d}, J=$ $8.5 \mathrm{~Hz}, 2 \mathrm{H}), 7.56(\mathrm{~d}, J=8.7 \mathrm{~Hz}, 2 \mathrm{H}), 6.99(\mathrm{~d}, J=8.7 \mathrm{~Hz}, 2 \mathrm{H}), 4.01$ $(\mathrm{t}, J=6.6 \mathrm{~Hz}, 2 \mathrm{H}), 2.67(\mathrm{~s}, 3 \mathrm{H}), 1.84-1.78(\mathrm{~m}, 2 \mathrm{H}), 1.51-1.45(\mathrm{~m}$, $2 \mathrm{H}), 1.40-1.26(\mathrm{~m}, 8 \mathrm{H}), 0.90(\mathrm{t}, J=6.9 \mathrm{~Hz}, 2 \mathrm{H}) \mathrm{ppm} .{ }^{13} \mathrm{C} \mathrm{NMR}$ $\left(125 \mathrm{MHz}, \mathrm{CDCl}_{3}\right): \delta=174.7,159.9,145.2,133.8,131.5,128.4$, 128.3, 127.1, 115.2, 113.2, 83.8, 68.3, 32.0, 29.5, 29.4, 29.4, 26.2, 24.1, 22.8, 14.3 ppm. HRMS (MALDI +ve) calcd for $\mathrm{C}_{25} \mathrm{H}_{28} \mathrm{~N}_{2} \mathrm{ONa}\left[(\mathrm{M}+\mathrm{Na})^{+}\right]: m / z=395.2094 ; \exp 395.2102$. Analysis calcd (\%) for $\mathrm{C}_{25} \mathrm{H}_{28} \mathrm{~N}_{2} \mathrm{O}$ (372.51): C 80.61, H 7.58, N 7.52; found: $\mathrm{C} 80.67, \mathrm{H} 7.67, \mathrm{~N} 7.38$.

2-(1-(2',3'-Difluoro-4'-(octyloxy)-[1,1'-biphenyl]-4-yl)ethylidene) malononitrile (13g). White solid. $R_{\mathrm{f}}=0.61$ (toluene). $\mathrm{Mp}=59.5-$ $60.4{ }^{\circ} \mathrm{C} .{ }^{1} \mathrm{H}$ NMR $\left(500 \mathrm{MHz}, \mathrm{CDCl}_{3}\right): \delta=7.65$ (apparent s, $4 \mathrm{H}$ ), 7.16-7.10 (ddd, $J=8.7,8.2,2.4,1 \mathrm{H}), 6.83(\mathrm{ddd}, J=9.1,7.5,1.8$ $\mathrm{Hz}, 1 \mathrm{H}), 4.09$ (t, J = 6.6 Hz, 2H), $2.68(\mathrm{~s}, 3 \mathrm{H}), 1.92-1.81(\mathrm{~m}, 2 \mathrm{H})$, 1.54-1.44 (m, 2H), 1.42-1.22 (m, 8H), $0.89(\mathrm{t}, J=6.8,1 \mathrm{H}) \mathrm{ppm}$. ${ }^{13} \mathrm{C} \mathrm{NMR}\left(125 \mathrm{MHz}, \mathrm{CDCl}_{3}\right): \delta=174.7,149.1$ (dd, $J=250,11.4$ $\mathrm{Hz}), 149.0$ (dd, $J=8.1,3.1 \mathrm{~Hz}), 141.9(\mathrm{dd}, J=248.2,14.8 \mathrm{~Hz}$ ), $139.3,134.9,129.4,129.4,127.9$, 123.8, 123.7, 123.7, 121.2, 121.1, 113.1, 112.9, 109.9, 84.7, 70.1, 31.9, 29.4, 29.4, 29.3, 26.0, 24.3, 22.8, 14.2 ppm. HRMS (MALDI +ve) calcd for $\mathrm{C}_{25} \mathrm{H}_{27} \mathrm{~F}_{2} \mathrm{~N}_{2} \mathrm{O}$ $\left[(\mathrm{M}+\mathrm{H})^{+}\right]: m / z=409.2086$; $\exp 409.2095$. Analysis calcd (\%) for $\mathrm{C}_{25} \mathrm{H}_{26} \mathrm{~F}_{2} \mathrm{~N}_{2} \mathrm{O}$ (408.49): C 73.51, $\mathrm{H} 6.42$, N 6.86; found: $\mathrm{C} 73.33$, H 6.30, N 6.78. 
2-(1-(2,3-Difluoro-4-(octyloxy)phenyl)ethylidene)malononitrile (13h). Off-white solid. $R_{\mathrm{f}}=0.62$ (toluene); $\mathrm{Mp}=59.1-60.5{ }^{\circ} \mathrm{C} ;{ }^{1} \mathrm{H}$ $\operatorname{NMR}\left(500 \mathrm{MHz}, \mathrm{CDCl}_{3}\right): \delta=7.13(\mathrm{ddd}, J=9.9,7.6,2.2 \mathrm{~Hz}, 1 \mathrm{H})$, 6.82 (ddd, $J=9.9,7.2,1.8 \mathrm{~Hz}, 1 \mathrm{H}), 4.09(\mathrm{t}, J=6.5 \mathrm{~Hz}, 2 \mathrm{H}), 2.61$ $(\mathrm{d}, J=1.6 \mathrm{~Hz}, 3 \mathrm{H}), 1.92-1.74(\mathrm{~m}, 2 \mathrm{H}), 1.50-1.39(\mathrm{~m}, 2 \mathrm{H}), 1.41-$ $1.19(\mathrm{~m}, 8 \mathrm{H}), 0.94-0.76(\mathrm{~m}, 3 \mathrm{H}) \mathrm{ppm} .{ }^{13} \mathrm{C}$ NMR $(125 \mathrm{MHz}$, $\left.\mathrm{CDCl}_{3}\right): \delta=170.3(\mathrm{dd}, J=2.5,1.5 \mathrm{~Hz}), 151.9(\mathrm{dd}, J=8.1,3.7 \mathrm{~Hz})$, 148.5 (dd, $J=254.3,12.1 \mathrm{~Hz}$ ), 141.6 , (dd, $J=251.7,13.8 \mathrm{~Hz}$ ), $123.2(\mathrm{dd}, J=4.5,3.2 \mathrm{~Hz}), 117.4(\mathrm{~d}, J=10.6 \mathrm{~Hz}), 112.2,112.1$, 109.3 (dd, $J=3.1,1.3 \mathrm{~Hz}$ ), 87.3, 70.1, 31.8, 29.2, 29.2, 28.9, 25.8, $24.1(\mathrm{~d}, J=4.6 \mathrm{~Hz}), 22.7,14.1 \mathrm{ppm}$; HRMS (MALDI +ve) calcd for $\mathrm{C}_{19} \mathrm{H}_{22} \mathrm{~F}_{2} \mathrm{~N}_{2} \mathrm{ONa}\left[(\mathrm{M}+\mathrm{Na})^{+}\right]: \mathrm{m} / \mathrm{z}: 355.1592$, found $\mathrm{m} / \mathrm{z}=$ 355.1600 .

2-(1-(4-Octylphenyl)ethylidene)malononitrile (13i). White solid. $R_{\mathrm{f}}=0.65$ (toluene). $\mathrm{Mp}=51.5-52.0{ }^{\circ} \mathrm{C} .{ }^{1} \mathrm{H}$ NMR $(500 \mathrm{MHz}$, $\left.\mathrm{CDCl}_{3}\right): \delta=7.50(\mathrm{~d}, J=8.3 \mathrm{~Hz}, 2 \mathrm{H}), 7.30(\mathrm{~d}, J=8.3 \mathrm{~Hz}, 2 \mathrm{H}), 2.66$ $(\mathrm{t}, J=7.7 \mathrm{~Hz}, 2 \mathrm{H}), 2.63(\mathrm{~s}, 3 \mathrm{H}), 1.66-1.1 .60(\mathrm{~m}, 2 \mathrm{H}), 1.35-1.27$ $(\mathrm{m}, 10 \mathrm{H}), 0.88(\mathrm{t}, J=7.1 \mathrm{~Hz}, 3 \mathrm{H}) \mathrm{ppm} .{ }^{13} \mathrm{C}$ NMR $(125 \mathrm{MHz}$, $\left.\mathrm{CDCl}_{3}\right): \delta=175.3,148.5,133.3,129.3,127.7,113.3,113.2,83.7$, $36.1,32.0,31.2,29.5,29.4,29.3,24.2,22.8,14.2 \mathrm{ppm}$. HRMS (MALDI +ve) calcd for $\mathrm{C}_{19} \mathrm{H}_{24} \mathrm{~N}_{2} \mathrm{Na}\left([\mathrm{M}+\mathrm{Na}]^{+}\right): m / z=303.1831$; exp 303.1838. Analysis calcd (\%) for $\mathrm{C}_{19} \mathrm{H}_{24} \mathrm{~N}_{2}$ (280.42): C 81.38, H 8.63, N 9.99; found: C 81.60, H 8.62, N 10.05 .

2-(4-((5-(4-Cyanophenoxy)pentyl)oxy)phenyl)azulene-1,1(8aH)dicarbonitrile (3a). To a solution of $13 \mathrm{a}(882 \mathrm{mg}, 2.37 \mathrm{mmol})$ and $\left[\mathrm{C}_{7} \mathrm{H}_{7}\right] \mathrm{BF}_{4}(483 \mathrm{mg}, 2.71 \mathrm{mmol})$ in $\mathrm{CH}_{2} \mathrm{Cl}_{2}(100 \mathrm{~mL})$ at -78 ${ }^{\circ} \mathrm{C}$, under an argon atmosphere, was slowly added $\mathrm{NEt}_{3}(0.70$ $\mathrm{mL}, 5.0 \mathrm{mmol}$ ) and the resulting solution stirred for $30 \mathrm{~min}$. The reaction was quenched by addition of aqueous $1 \mathrm{M} \mathrm{HCl}$ $(100 \mathrm{~mL})$ and the vessel allowed to reach ambient temperature. The phases were separated and the organic phase dried over $\mathrm{MgSO}_{4}$, filtered and the solvent removed under reduced pressure. The residue was dissolved in $\mathrm{CH}_{2} \mathrm{ClCH}_{2} \mathrm{Cl}(50 \mathrm{~mL})$ and to the vessel $\left[\mathrm{Ph}_{3} \mathrm{C}\right] \mathrm{BF}_{4}(882 \mathrm{mg}, 2.49 \mathrm{mmol})$ was added whereafter the mixture was heated to reflux point for $2 \mathrm{~h}$. The cooled vessel was placed in an ice bath and $\mathrm{NEt}_{3}(0.50 \mathrm{~mL}, 3.6 \mathrm{mmol})$ was added slowly to the flask. Toluene $(50 \mathrm{~mL})$ was added to the flask and the contents allowed to sit overnight at rt, after which time the solvent was removed in vacuo and the crude residue purified by flash column chromatography ( $2 \%$ EtOAc/toluene) to give $3 \mathrm{a}(345 \mathrm{mg}, 32 \%)$, as an orange solid. $R_{\mathrm{f}}=0.30(2 \%$ EtOAc/toluene). $\mathrm{Mp}=141.0-145.2{ }^{\circ} \mathrm{C} .{ }^{1} \mathrm{H}$ NMR $(500 \mathrm{MHz}$, $\left.\mathrm{CDCl}_{3}\right): \delta=7.70(\mathrm{~d}, J=8.9 \mathrm{~Hz}, 2 \mathrm{H}), 7.61(\mathrm{~d}, J=8.9 \mathrm{~Hz}, 2 \mathrm{H}), 6.99$ $(\mathrm{d}, J=8.9 \mathrm{~Hz}, 2 \mathrm{H}) ; 6.97(\mathrm{~d}, J=8.9 \mathrm{~Hz}, 2 \mathrm{H}), 6.79(\mathrm{~s}, 1 \mathrm{H}), 6.59(\mathrm{dd}$, $J=11.3,6.4 \mathrm{~Hz}, 1 \mathrm{H}), 6.47(\mathrm{dd}, J=11.3,6.1 \mathrm{~Hz}, 1 \mathrm{H}), 6.34-6.31$ $(\mathrm{m}, 2 \mathrm{H}), 5.84(\mathrm{dd}, J=10.2,3.9 \mathrm{~Hz}, 1 \mathrm{H}), 4.08(\mathrm{t}, J=6.3 \mathrm{~Hz}, 2 \mathrm{H})$, $4.07(\mathrm{t}, J=6.3 \mathrm{~Hz}, 2 \mathrm{H}), 3.80(\mathrm{ddd}, J=3.9,1.9,1.9 \mathrm{~Hz}, 1 \mathrm{H}), 1.96-$ $1.89(\mathrm{~m}, 4 \mathrm{H}), 1.74-1.69(\mathrm{~m}, 2 \mathrm{H}) \mathrm{ppm} .{ }^{13} \mathrm{C}$ NMR $(125 \mathrm{MHz}$, $\left.\mathrm{CDCl}_{3}\right): \delta=162.3,160.4,139.9,139.0,134.0,131.0,130.4$, $130.1,127.8,127.6,123.0,120.0,119.4,119.3,115.3,115.2$, 115.1, 112.9, 103.7, 68.1, 67.8, 51.1, 45.2, 28.8, 28.8, 22.7 ppm. HRMS (MALDI +ve) calcd for $\mathrm{C}_{30} \mathrm{H}_{26} \mathrm{~N}_{3} \mathrm{O}_{2}\left([\mathrm{M}+\mathrm{H}]^{+}\right): m / z=$ 460.2020; exp 460.2021. Analysis calcd (\%) for $\mathrm{C}_{30} \mathrm{H}_{25} \mathrm{~N}_{3} \mathrm{O}_{2}$ (459.55): C 78.41, H 5.48, N 9.14; found: C 78.11, H 5.40, N 9.05.

2-(4-((6-(4-Cyanophenoxy)hexyl)oxy)phenyl)azulene-1,1(8aH)dicarbonitrile (3b). To a solution of $\mathbf{1 3 b}(1.05 \mathrm{~g}, 2.72 \mathrm{mmol})$ and
$\left[\mathrm{C}_{7} \mathrm{H}_{7}\right] \mathrm{BF}_{4}(527 \mathrm{mg}, 2.96 \mathrm{mmol})$ in $\mathrm{CH}_{2} \mathrm{Cl}_{2}(100 \mathrm{~mL})$ at $-78{ }^{\circ} \mathrm{C}$, under an argon atmosphere, was slowly added $\mathrm{NEt}_{3}(1.0 \mathrm{~mL}, 7.2$ $\mathrm{mmol}$ ) and the resulting solution stirred for $30 \mathrm{~min}$. The reaction was quenched by addition of aqueous $1 \mathrm{M} \mathrm{HCl}(100 \mathrm{~mL})$ and the vessel allowed to reach ambient temperature. The phases were separated and the organic phase dried over $\mathrm{MgSO}_{4}$, filtered and the solvent removed under reduced pressure. The residue was dissolved in $\mathrm{CH}_{2} \mathrm{ClCH}_{2} \mathrm{Cl}(50 \mathrm{~mL})$ and to the vessel $\left[\mathrm{Ph}_{3} \mathrm{C}\right] \mathrm{BF}_{4}(1.00 \mathrm{~g}, 3.03 \mathrm{mmol})$ was added whereafter the mixture was heated to reflux point for $2 \mathrm{~h}$. The cooled vessel was placed in an ice bath and $\mathrm{NEt}_{3}(0.70 \mathrm{~mL}, 5.0 \mathrm{mmol})$ was added slowly to the flask. Toluene $(50 \mathrm{~mL})$ was added to the flask and the contents allowed to sit overnight at rt, after which time the solvent was removed in vacuo and the crude residue purified by flash column chromatography ( $2 \%$ EtOAc/toluene) to give $\mathbf{3 b}$ (603 $\mathrm{mg}, 47 \%)$, as a fluffy yellow solid. $R_{\mathrm{f}}=0.33(2 \% \mathrm{EtOAc} /$ toluene). $\mathrm{Mp}=122.1-124.0{ }^{\circ} \mathrm{C} .{ }^{1} \mathrm{H}$ NMR $\left(500 \mathrm{MHz}, \mathrm{CDCl}_{3}\right)$ : $\delta=7.67(\mathrm{~d}, J=8.9 \mathrm{~Hz}, 2 \mathrm{H}), 7.57(\mathrm{~d}, J=8.9 \mathrm{~Hz}, 2 \mathrm{H}), 6.96(\mathrm{~d}, J=$ $8.9 \mathrm{~Hz}, 2 \mathrm{H}), 6.93(\mathrm{~d}, J=8.9 \mathrm{~Hz}, 2 \mathrm{H}), 6.75(\mathrm{~s}, 1 \mathrm{H}), 6.55(\mathrm{dd}, J=$ $11.3,6.4 \mathrm{~Hz}, 1 \mathrm{H}), 6.44(\mathrm{dd}, J=11.3,6.1 \mathrm{~Hz}, 1 \mathrm{H}), 6.31-6.28(\mathrm{~m}$, $2 \mathrm{H}), 5.81(\mathrm{dd}, J=10.2,3.9 \mathrm{~Hz}, 1 \mathrm{H}), 4.03(\mathrm{t}, J=6.4 \mathrm{~Hz}, 2 \mathrm{H}), 4.02$ $(\mathrm{t}, J=6.4 \mathrm{~Hz}, 2 \mathrm{H}), 3.77$ (ddd, $J=3.9,2.0,2.0 \mathrm{~Hz}, 1 \mathrm{H}), 1.88-1.82$ $(\mathrm{m}, 3 \mathrm{H}), 1.57-1.54(\mathrm{~m}, 3 \mathrm{H}) \mathrm{ppm} .{ }^{13} \mathrm{C}$ NMR $\left(125 \mathrm{MHz}, \mathrm{CDCl}_{3}\right): \delta=$ $162.5,160.6,140.1,139.2,134.1,131.1,130.5,130.2,128.0$, 127.8, 123.1, 120.1, 119.4, 119.4, 115.3, 115.3, 113.0, 68.3, 68.1, 51.3, 45.4, 29.2, 29.1, 25.9, 25.9 ppm, 1C masked. HRMS (MALDI +ve) calcd for $\mathrm{C}_{31} \mathrm{H}_{28} \mathrm{~N}_{3} \mathrm{O}_{2}\left([\mathrm{M}+\mathrm{H}]^{+}\right): m / z=474.2176$; $\exp$ 474.2176. Analysis calcd (\%) for $\mathrm{C}_{31} \mathrm{H}_{27} \mathrm{~N}_{3} \mathrm{O}_{2}$ (473.58): $\mathrm{C} 78.62, \mathrm{H}$ 5.75, N 8.87; found: C 78.31, H 5.42, N 8.69.

2-(4-((8-(4-Cyanophenoxy)octyl)oxy)phenyl)azulene-1,1(8aH)dicarbonitrile (3c). To a solution of $13 \mathrm{c}(1.04 \mathrm{~g}, 2.52 \mathrm{mmol})$ and $\left[\mathrm{C}_{7} \mathrm{H}_{7}\right] \mathrm{BF}_{4}(522 \mathrm{mg}, 2.93 \mathrm{mmol})$ in $\mathrm{CH}_{2} \mathrm{Cl}_{2}(100 \mathrm{~mL})$ at $-78^{\circ} \mathrm{C}$, under an argon atmosphere, was slowly added $\mathrm{NEt}_{3}(1.0 \mathrm{~mL}$, $7.2 \mathrm{mmol}$ ) and the resulting solution stirred for $30 \mathrm{~min}$. The reaction was quenched by addition of aqueous $1 \mathrm{M} \mathrm{HCl} \mathrm{(100}$ $\mathrm{mL}$ ) and the vessel allowed to reach ambient temperature. The phases were separated and the organic phase dried over $\mathrm{MgSO}_{4}$, filtered and the solvent removed under reduced pressure. The residue was dissolved in $\mathrm{CH}_{2} \mathrm{ClCH}_{2} \mathrm{Cl}(50 \mathrm{~mL})$ and to the vessel $\left[\mathrm{Ph}_{3} \mathrm{C}\right] \mathrm{BF}_{4}(929 \mathrm{mg}, 2.81 \mathrm{mmol})$ was added whereafter the mixture was heated to reflux point for $2 \mathrm{~h}$. The cooled vessel was placed in an ice bath and $\mathrm{NEt}_{3}(0.70 \mathrm{~mL}, 5.0$ $\mathrm{mmol})$ was added slowly to the flask. Toluene $(50 \mathrm{~mL})$ was added to the flask and the contents allowed to sit overnight at rt, after which time the solvent was removed in vacuo and the crude residue purified by flash column chromatography $(2 \%$ EtOAc/toluene) to give $3 \mathrm{c}(200 \mathrm{mg}, 16 \%)$ as an orange solid. $R_{\mathrm{f}}$ $=0.35$ (2\% EtOAc/toluene). $\mathrm{Mp}=98.5-101.2{ }^{\circ} \mathrm{C} .{ }^{1} \mathrm{H}$ NMR $(500$ $\left.\mathrm{MHz}, \mathrm{CDCl}_{3}\right): \delta=7.67(\mathrm{~d}, J=8.9 \mathrm{~Hz}, 2 \mathrm{H}), 7.57(\mathrm{~d}, J=8.9 \mathrm{~Hz}$, $2 \mathrm{H}), 6.96(\mathrm{~d}, J=8.9 \mathrm{~Hz}, 2 \mathrm{H}), 6.93(\mathrm{~d}, J=8.9 \mathrm{~Hz}, 2 \mathrm{H}), 6.75(\mathrm{~s}$, $1 \mathrm{H}), 6.55(\mathrm{dd}, J=11.2,6.4 \mathrm{~Hz}, 1 \mathrm{H}), 6.44(\mathrm{dd}, J=11.3,6.1 \mathrm{~Hz}$, $1 \mathrm{H}), 6.31-6.28(\mathrm{~m}, 2 \mathrm{H}), 5.81(\mathrm{dd}, J=10.2,3.8 \mathrm{~Hz}, 1 \mathrm{H}), 4.01(\mathrm{t}, J$ $=6.5 \mathrm{~Hz}, 1 \mathrm{H}), 4.00(\mathrm{~d}, J=6.5 \mathrm{~Hz}, 1 \mathrm{H}), 3.77(\mathrm{ddd}, J=3.9,2.0$, $2.0 \mathrm{~Hz}, 1 \mathrm{H}), 1.84-1.78(\mathrm{~m}, 4 \mathrm{H}), 1.51-1.45(\mathrm{~m}, 4 \mathrm{H}), 1.42-1.39$ (m, 4H) ppm. ${ }^{13} \mathrm{C}$ NMR $\left(125 \mathrm{MHz}, \mathrm{CDCl}_{3}\right): \delta=162.6,160.7$, 140.2, 139.2, 134.1, 131.1, 130.4, 130.2, 127.9, 127.8, 123.0, 120.0, 119.5, 119.4, 115.5, 115.3, 113.0, 103.8, 68.5, 68.3, 51.3, 
45.4, 29.4, 29.2, 29.1, 26.1, 26.0 ppm, 2C masked. HRMS (MALDI +ve) calcd for $\mathrm{C}_{33} \mathrm{H}_{32} \mathrm{~N}_{3} \mathrm{O}_{2}\left([\mathrm{M}+\mathrm{H}]^{+}\right): m / z=502.2489$; $\exp 502.2490$.

2-(4-((9-(4-Cyanophenoxy)nonyl)oxy)phenyl)azulene-1,1(8aH)dicarbonitrile (3d). To a solution of $13 \mathrm{~d}(1.02 \mathrm{~g}, 2.39 \mathrm{mmol})$ and $\left[\mathrm{C}_{7} \mathrm{H}_{7}\right] \mathrm{BF}_{4}(500 \mathrm{mg}, 2.81 \mathrm{mmol})$ in $\mathrm{CH}_{2} \mathrm{Cl}_{2}(100 \mathrm{~mL})$ at $-78{ }^{\circ} \mathrm{C}$, under an argon atmosphere, was slowly added $\mathrm{NEt}_{3}(1.0 \mathrm{~mL}$, $7.2 \mathrm{mmol}$ ) and the resulting solution stirred for $30 \mathrm{~min}$. The reaction was quenched by addition of aqueous $1 \mathrm{M} \mathrm{HCl}(100$ $\mathrm{mL}$ ) and the vessel allowed to reach ambient temperature. The phases were separated and the organic phase dried over $\mathrm{MgSO}_{4}$, filtered and the solvent removed under reduced pressure. The residue was dissolved in $\mathrm{CH}_{2} \mathrm{ClCH}_{2} \mathrm{Cl}(50 \mathrm{~mL})$ and to the vessel $\left[\mathrm{Ph}_{3} \mathrm{C}\right] \mathrm{BF}_{4}(935 \mathrm{mg}, 2.83 \mathrm{mmol})$ was added whereafter the mixture was heated to reflux point for $2 \mathrm{~h}$. The cooled vessel was placed in an ice bath and $\mathrm{NEt}_{3}(0.70 \mathrm{~mL}, 5.0 \mathrm{mmol})$ was added slowly to the flask. Toluene $(50 \mathrm{~mL})$ was added to the flask and the contents allowed to sit overnight at rt, after which time the solvent was removed in vacuo and the crude residue purified by flash column chromatography (2\% EtOAc/ toluene) to give $3 \mathbf{d}(258 \mathrm{mg}, 21 \%)$ as an orange solid. $R_{\mathrm{f}}=0.36$ (2\% EtOAc/toluene). $\mathrm{Mp}=98.9-103.8,125.6-130.6{ }^{\circ} \mathrm{C} .{ }^{1} \mathrm{H}$ NMR (500 MHz, $\left.\mathrm{CDCl}_{3}\right): \delta 7.67(\mathrm{~d}, J=8.8 \mathrm{~Hz}, 2 \mathrm{H}), 7.57(\mathrm{~d}, J=$ $8.8 \mathrm{~Hz}, 2 \mathrm{H}), 6.96(\mathrm{~d}, J=8.8 \mathrm{~Hz}, 2 \mathrm{H}), 6.93(\mathrm{~d}, J=8.8 \mathrm{~Hz}, 2 \mathrm{H})$, $6.75(\mathrm{~s}, 1 \mathrm{H}), 6.55(\mathrm{dd}, J=11.2,6.4 \mathrm{~Hz}, 1 \mathrm{H}), 6.44(\mathrm{dd}, J=11.2$, $6.1 \mathrm{~Hz}, 1 \mathrm{H}), 6.31-6.28(\mathrm{~m}, 2 \mathrm{H}), 5.81(\mathrm{dd}, J=10.2,3.8 \mathrm{~Hz}, 1 \mathrm{H})$, $4.01(\mathrm{t}, J=6.8 \mathrm{~Hz}, 2 \mathrm{H}), 4.00(\mathrm{t}, J=6.8 \mathrm{~Hz}, 2 \mathrm{H}), 3.77$ (ddd, $J=$ $3.8,2.0,2.0 \mathrm{~Hz}, 1 \mathrm{H}), 1.83-1.78(\mathrm{~m}, 4 \mathrm{H}), 1.49-1.44(\mathrm{~m}, 4 \mathrm{H})$, 1.40-1.37 (m, 6H) ppm. $\left.{ }^{13} \mathrm{C} \mathrm{NMR} \mathrm{(125} \mathrm{MHz,} \mathrm{CDCl}_{3}\right): \delta=162.6$, $160.7,140.2$, 139.2, 134.1, 131.1, 130.4, 130.2, 127.9, 127.8, 123.0, 120.0, 119.5, 119.4, 115.5, 115.3, 113.0, 103.8, 68.5, 68.3, 51.3, 45.4, 29.6, 29.4, 29.2, 29.1, 26.1, 26.1 ppm, 2Cs masked. HRMS (MALDI +ve) calcd for $\mathrm{C}_{34} \mathrm{H}_{34} \mathrm{~N}_{3} \mathrm{O}_{2}\left([\mathrm{M}+\mathrm{H}]^{+}\right): m / z=$ 516.2646; exp 516.2649. Analysis calcd (\%) for $\mathrm{C}_{34} \mathrm{H}_{33} \mathrm{~N}_{3} \mathrm{O}_{2}$ (515.66): C 79.19, H 6.45, N 8.15; found: C 78.68, H 6.18, N 8.11 .

2-(4-((10-(4-Cyanophenoxy)decyl)oxy)phenyl)azulene-1,1(8aH)dicarbonitrile (3e). To a solution of $13 \mathrm{e}(1.12 \mathrm{~g}, 2.54 \mathrm{mmol})$ and $\left[\mathrm{C}_{7} \mathrm{H}_{7}\right] \mathrm{BF}_{4}(503 \mathrm{mg}, 2.83 \mathrm{mmol})$ in $\mathrm{CH}_{2} \mathrm{Cl}_{2}(100 \mathrm{~mL})$ at $-78{ }^{\circ} \mathrm{C}$, under an argon atmosphere, was slowly added $\mathrm{NEt}_{3}(1.0 \mathrm{~mL}$, $7.2 \mathrm{mmol}$ ) and the resulting solution stirred for $30 \mathrm{~min}$. The reaction was quenched by addition of aqueous $1 \mathrm{M} \mathrm{HCl} \mathrm{(100}$ $\mathrm{mL}$ ) and the vessel allowed to reach ambient temperature. The phases were separated and the organic phase dried over $\mathrm{MgSO}_{4}$, filtered and the solvent removed under reduced pressure. The residue was dissolved in $\mathrm{CH}_{2} \mathrm{ClCH}_{2} \mathrm{Cl}(50 \mathrm{~mL})$ and to the vessel $\left[\mathrm{Ph}_{3} \mathrm{C}\right] \mathrm{BF}_{4}(851 \mathrm{mg}, 2.58 \mathrm{mmol})$ was added whereafter the mixture was heated to reflux point for $2 \mathrm{~h}$. The cooled vessel was placed in an ice bath and $\mathrm{NEt}_{3}(0.70 \mathrm{~mL}, 5.0 \mathrm{mmol})$ was added slowly to the flask. Toluene $(50 \mathrm{~mL})$ was added to the flask and the contents allowed to sit overnight at rt, after which time the solvent was removed in vacuo and the crude residue purified by flash column chromatography ( $2 \%$ EtOAc/toluene) to give $3 \mathrm{e}(552 \mathrm{mg}, 41 \%)$, as an orange solid. $R_{\mathrm{f}}=0.38(2 \%$ EtOAc/toluene). $\mathrm{Mp}=102.2-121.8{ }^{\circ} \mathrm{C} .{ }^{1} \mathrm{H} \mathrm{NMR}(500 \mathrm{MHz}$, $\left.\mathrm{CDCl}_{3}\right): \delta=7.67(\mathrm{~d}, J=8.9 \mathrm{~Hz}, 2 \mathrm{H}), 7.57(\mathrm{~d}, J=8.9 \mathrm{~Hz}, 2 \mathrm{H}), 6.97$ $(\mathrm{d}, J=8.9 \mathrm{~Hz}, 2 \mathrm{H}), 6.93$ (d, $J=8.9 \mathrm{~Hz}, 2 \mathrm{H}), 6.75$ (s, 1H), 6.55 (dd,
$J=11.3,6.3 \mathrm{~Hz}, 1 \mathrm{H}), 6.44(\mathrm{dd}, J=11.3,6.1 \mathrm{~Hz}, 1 \mathrm{H}), 6.31-6.28$ $(\mathrm{m}, 2 \mathrm{H}), 5.81(\mathrm{dd}, J=10.2,3.8 \mathrm{~Hz}, 1 \mathrm{H}), 4.01(\mathrm{~d}, J=6.6 \mathrm{~Hz}, 2 \mathrm{H})$, $3.99(\mathrm{~d}, J=6.6 \mathrm{~Hz}, 2 \mathrm{H}), 3.77$ (ddd, $J=3.8,1.9,1.9 \mathrm{~Hz}, 1 \mathrm{H}), 1.83-$ 1.77 (m, 4H), 1.49-1.43 (m, 4H), 1.39-1.32 (m, 6H) ppm. ${ }^{13} \mathrm{C}$ NMR (125 MHz, $\left.\mathrm{CDCl}_{3}\right): \delta=162.6,160.7,140.2,139.2,134.1$, $131.1,130.4,130.1,127.9,127.8,123.0,120.0,119.5,119.4$, $115.5,115.3,115.3,113.0,103.8,68.5,68.3,51.3,45.4,29.6$, 29.6, 29.5 , 29.5, 29.2, 29.1, 26.1, 26.1 ppm. HRMS (MALDI +ve) calcd for $\mathrm{C}_{35} \mathrm{H}_{36} \mathrm{~N}_{3} \mathrm{O}_{2}\left([\mathrm{M}+\mathrm{H}]^{+}\right): m / z=530.2802$; $\exp 530.2806$.

2-(4'-(Octyloxy)-[1,1'-biphenyl]-4-yl)azulene-1,1(8aH)-dicarbonitrile (3f). Method 1: to a solution/suspension of the crude mixture from $13 f(3.54 \mathrm{~g}, 9.50 \mathrm{mmol})$ and $\left[\mathrm{C}_{7} \mathrm{H}_{7}\right] \mathrm{BF}_{4}(1.71 \mathrm{~g}, 9.61$ $\mathrm{mmol})$ in $\mathrm{CH}_{2} \mathrm{Cl}_{2}(250 \mathrm{~mL})$, at $-78{ }^{\circ} \mathrm{C}$ under an argon atmosphere, was added $\mathrm{NEt}_{3}(1.0 \mathrm{~g}, 1.4 \mathrm{~mL}, 10 \mathrm{mmol})$ over a $30 \mathrm{~min}$ period and the reaction mixture stirred at $-78{ }^{\circ} \mathrm{C}$ for $2 \mathrm{~h}$. Additional $\mathrm{NEt}_{3}$ was added $(0.5 \mathrm{~mL}, 3.58 \mathrm{mmol})$ and the reaction mixture was left to stir for $1 \mathrm{~h}$ and allowed to reach $\mathrm{rt}$ and subjected to ultrasonication. $\mathrm{HCl}(150 \mathrm{~mL}, 2 \mathrm{M})$ was added to the vessel and the phases separated. The aqueous phase was extracted with $\mathrm{CH}_{2} \mathrm{Cl}_{2}(100 \mathrm{~mL})$ and the combined organic phases dried over $\mathrm{MgSO}_{4}$, filtered, and the solvent was removed in vacuo. The residue was taken up in $\mathrm{CH}_{2} \mathrm{ClCH}_{2} \mathrm{Cl}(125 \mathrm{~mL})$ and treated with $\left[\mathrm{Ph}_{3} \mathrm{C}\right] \mathrm{BF}_{4}(3.85 \mathrm{~g}, 11.6 \mathrm{mmol})$ and subjected to reflux for $2 \mathrm{~h}$ under inert atmosphere. The reaction mixture was diluted with toluene $(125 \mathrm{~mL})$ and cooled to $0{ }^{\circ} \mathrm{C}$ and $\mathrm{NEt}_{3}(1.2 \mathrm{~g}$, $1.7 \mathrm{~mL}, 12 \mathrm{mmol}$ ) was added, where the mixture was stirred overnight and allowed to reach room temperature. The solvent was removed in vacuum, and the residue was subjected to flash chromatography (40-50\% $\mathrm{CH}_{2} \mathrm{Cl}_{2}$ /heptane) and crystallized from $\mathrm{CH}_{2} \mathrm{Cl}_{2}$ /ethanol to furnish $3 f(502 \mathrm{mg}, 11 \%)$ as a yellow solid. Method 2: To a degassed solution of 15 (1.54 g, $4.03 \mathrm{mmol})$ in toluene $(60 \mathrm{~mL})$ and water $(15 \mathrm{~mL})$ was added $\mathrm{Pd}(\mathrm{OAc})_{2}(55$ $\mathrm{mg}, 0.245 \mathrm{mmol}$ ), RuPhos (187 mg, $0.400 \mathrm{mmol}), \mathrm{K}_{3} \mathrm{PO}_{4}(1.31 \mathrm{~g}$, $6.17 \mathrm{mmol})$ and $11 \mathrm{f}(1.56 \mathrm{~g}, 6.24 \mathrm{mmol})$, and the biphasic mixture stirred for $18 \mathrm{~h}$ at $80{ }^{\circ} \mathrm{C}$. TLC indicated an incomplete reaction and more $\mathrm{Pd}(\mathrm{OAc})_{2}(60 \mathrm{mg}, 0.267 \mathrm{mmol})$, RuPhos (195 $\mathrm{mg}, 0.419 \mathrm{mmol})$ and $\mathrm{K}_{3} \mathrm{PO}_{4}(2.32 \mathrm{~g}, 10.9 \mathrm{mmol})$ and $11 \mathrm{f}(1.21 \mathrm{~g}$, $\mathrm{mmol}$ ) were added and the mixture heated to $80{ }^{\circ} \mathrm{C}$ for another $18 \mathrm{~h}$. The contents of the vessel were diluted with water (100 $\mathrm{mL}$ ) and the phases were separated. The aqueous phase was extracted with $\mathrm{CH}_{2} \mathrm{Cl}_{2}(100 \mathrm{~mL})$ and the combined organic extracts were dried over $\mathrm{MgSO}_{4}$, filtered and the solvent removed in vacuo. The crude residue was subjected to flash column chromatography (gradient elution of 55-70\% toluene/ heptane) and subsequently recrystallized from $\mathrm{CH}_{2} \mathrm{Cl}_{2}$ / heptane to give pure 3f (562 mg, 30\%). $R_{\mathrm{f}}=0.42\left(50 \% \mathrm{CH}_{2} \mathrm{Cl}_{2} /\right.$ heptane). $\mathrm{Mp}=\mathrm{Cr} 90.6$ (N 75.7) Iso; Iso $75.6 \mathrm{~N} 29.8 T_{\mathrm{g}}{ }^{\circ} \mathrm{C} .{ }^{1} \mathrm{H}$ NMR $\left(500 \mathrm{MHz}, \mathrm{CDCl}_{3}\right): \delta=7.78(\mathrm{~d}, J=8.3 \mathrm{~Hz}, 2 \mathrm{H}), 7.66(\mathrm{~d}, J=$ $8.3 \mathrm{~Hz}, 2 \mathrm{H}), 7.56$ (d, $J=8.7 \mathrm{~Hz}, 2 \mathrm{H}), 6.99(\mathrm{~d}, J=8.7 \mathrm{~Hz}, 2 \mathrm{H}), 6.91$ $(\mathrm{s}, 1 \mathrm{H}), 6.57(\mathrm{dd}, J=11.2,6.4 \mathrm{~Hz}, 1 \mathrm{H}), 6.47(\mathrm{dd}, J=11.2,6.1 \mathrm{~Hz}$, $1 \mathrm{H}), 6.35(\mathrm{~d}, J=6.4 \mathrm{~Hz}, 1 \mathrm{H}), 6.31$ (ddd, $J=10.1,6.1,2.1 \mathrm{~Hz}, 1 \mathrm{H})$, $5.84(\mathrm{dd}, J=10.1,3.8 \mathrm{~Hz}, 1 \mathrm{H}), 4.01$ (t, $J=6.6 \mathrm{~Hz}, 1 \mathrm{H}), 3.81$ (ddd, $J=3.7,2.0,2.0 \mathrm{~Hz}, 1 \mathrm{H}), 1.84-1.78$ (m, 2H), 1.51-1.46 (m, 2H), 1.43-1.14 (m, 10H), $0.89(\mathrm{t}, J=6.9 \mathrm{~Hz}, 2 \mathrm{H}) \mathrm{ppm} .{ }^{13} \mathrm{C} \mathrm{NMR}(125$ $\left.\mathrm{MHz}_{\mathrm{CDCl}}\right): \delta=159.3,142.4,140.0,138.9,132.0,131.7,131.0$, $130.8,128.5,128.1,127.7,127.2$, 126.7, 120.7, 119.5, 115.2, $115.0,112.8,68.2,51.2,45.1,31.8,29.4,29.3,29.3,26.1,22.7$, 
14.1 ppm. HRMS (MALDI +ve) calcd for $\mathrm{C}_{32} \mathrm{H}_{32} \mathrm{~N}_{2} \mathrm{ONa}[(\mathrm{M}+$ $\mathrm{Na})^{+}$]: $m / z=483.2407$; $\exp 483.2406$. Analysis calcd (\%) for $\mathrm{C}_{32} \mathrm{H}_{32} \mathrm{~N}_{2} \mathrm{O}$ (460.62): C 83.44, $\mathrm{H} 7.00, \mathrm{~N} 6.08$; found: $\mathrm{C} 83.28, \mathrm{H}$ 6.90, N 6.12.

2-(2',3'-Difluoro-4'-(octyloxy)-[1,1'-biphenyl]-4-yl)azulene-1,1(8aH)dicarbonitrile ( $3 \mathrm{~g})$. Method 1: to a mixture of $13 \mathrm{~g}(3.35 \mathrm{~g}, 8.21 \mathrm{mmol})$ and $\left[\mathrm{C}_{7} \mathrm{H}_{7}\right] \mathrm{BF}_{4}(1.53 \mathrm{~g}, 8.60 \mathrm{mmol})$ in $\mathrm{CH}_{2} \mathrm{Cl}_{2}(250 \mathrm{~mL})$ at $-78{ }^{\circ} \mathrm{C}$ under argon atmosphere was added $\mathrm{NEt}_{3}(1.2 \mathrm{~mL}, 8.6 \mathrm{mmol})$ portion-wise over $30 \mathrm{~min}$ and the reaction mixture stirred at -78 ${ }^{\circ} \mathrm{C}$ for $2 \mathrm{~h}$. Since there were still starting material and tropylium left unreacted, more $\mathrm{NEt}_{3}$ was added $(0.5 \mathrm{~mL}, 3.58 \mathrm{mmol})$ and the reaction mixture was left to stir for $1 \mathrm{~h}$ more and allowed to reach $\mathrm{rt}$ while being sonicated. Then it was quenched with $\mathrm{HCl}(100 \mathrm{~mL}, 2$ $\mathrm{M})$ and diluted with water $(50 \mathrm{~mL})$. Subsequently, the phases were separated and the water phase was extracted with $\mathrm{CH}_{2} \mathrm{Cl}_{2}(100 \mathrm{~mL})$ and the combined organic phase was dried over $\mathrm{MgSO}_{4}$ and the solvent was removed by rotary evaporation. The residue was treated with $\left[\mathrm{Ph}_{3} \mathrm{C}\right] \mathrm{BF}_{4}(3.27 \mathrm{~g}, 9.89 \mathrm{mmol})$ in DCE $(125 \mathrm{~mL})$ under reflux for $2 \mathrm{~h}$ under an argon atmosphere, after which time the reaction mixture was cooled to $0{ }^{\circ} \mathrm{C}$ and diluted with toluene $(125$ $\mathrm{mL})$. When the reaction mixture reached $0{ }^{\circ} \mathrm{C}, \mathrm{NEt}_{3}(1.5 \mathrm{~mL}, 10$ mmol) was added, and the mixture was stirred overnight, while allowing to reach $\mathrm{rt}$, and left to stir for $15 \mathrm{~h}$. The solvent was removed in vacuo and the residue was subjected to flash column chromatography (40-50\% $\mathrm{CH}_{2} \mathrm{Cl}_{2} /$ heptane) and crystallized from $\mathrm{CH}_{2} \mathrm{Cl}_{2}$ and ethanol to give $3 \mathrm{~g}(1.104 \mathrm{~g}, 25 \%)$ as a yellow solid. Method 2: To a degassed solution of $15(2.05 \mathrm{~g}, 5.36 \mathrm{mmol})$ in toluene $(60 \mathrm{~mL})$ and water $(15 \mathrm{~mL})$ were added $\mathrm{Pd}(\mathrm{OAc})_{2}(76 \mathrm{mg}$, $0.339 \mathrm{mmol}$ ), RuPhos (262 $\mathrm{mg}, 0.561 \mathrm{mmol})$ and $\mathrm{K}_{3} \mathrm{PO}_{4}(2.51 \mathrm{~g}$, $11.8 \mathrm{mmol})$ and $11 \mathrm{~g}(1.56 \mathrm{~g}, \mathrm{mmol})$. Degassed water $(15 \mathrm{~mL})$ was added and the biphasic mixture stirred for $18 \mathrm{~h}$ at $90{ }^{\circ} \mathrm{C}$. Additional $\mathrm{Pd}(\mathrm{OAc})_{2}(62 \mathrm{mg}, 0.276 \mathrm{mmol})$, RuPhos $(206 \mathrm{mg}, 0.441$ $\mathrm{mmol})$ and $\mathrm{K}_{3} \mathrm{PO}_{4}(1.80 \mathrm{~g}, 8.48 \mathrm{mmol})$ and $11 \mathrm{~g}(1.04 \mathrm{~g}, \mathrm{mmol})$ were added and the mixture heated to $80{ }^{\circ} \mathrm{C}$ for a further $18 \mathrm{~h}$. The contents of the vessel were diluted with water $(100 \mathrm{~mL})$ and the phases were separated. The aqueous phase was extracted with $\mathrm{CH}_{2} \mathrm{Cl}_{2}(100 \mathrm{~mL})$ and the combined organic components were dried over $\mathrm{MgSO}_{4}$, filtered and the solvent removed in vacuo. The crude residue was subjected to flash column chromatography (20\% EtOAc/heptane) and a second flash column (gradient eluent $40-60 \% \mathrm{CH}_{2} \mathrm{Cl}_{2}$ /heptane) and then recrystallized from $\mathrm{CH}_{2} \mathrm{Cl}_{2} /$ heptane to give $3 \mathrm{~g}(522 \mathrm{mg}, 20 \%) . R_{\mathrm{f}}=0.44\left(50 \% \mathrm{CH}_{2} \mathrm{Cl}_{2} /\right.$ heptane $)$. $\mathrm{Mp}=\mathrm{Cr} 79.9$ (N 52.6) Iso; Iso $52.6 \mathrm{~N} 30.8 T_{\mathrm{g}}{ }^{\circ} \mathrm{C} .{ }^{1} \mathrm{H} \mathrm{NMR}(500 \mathrm{MHz}$, $\left.\mathrm{CDCl}_{3}\right): \delta=7.80(\mathrm{~d}, J=8.4 \mathrm{~Hz}, 2 \mathrm{H}), 7.62(\mathrm{dd}, J=8.4,1.3 \mathrm{~Hz}, 2 \mathrm{H})$, $7.13(\mathrm{td}, J=8.5,2.3 \mathrm{~Hz}, 1 \mathrm{H}), 6.93(\mathrm{~s}, 1 \mathrm{H}), 6.83(\mathrm{td}, J=8.5,1.6 \mathrm{~Hz}$, $1 \mathrm{H}), 6.58(\mathrm{dd}, J=11.2,6.3 \mathrm{~Hz}, 1 \mathrm{H}), 6.49(\mathrm{dd}, J=11.2,6.1 \mathrm{~Hz}, 1 \mathrm{H})$, $6.37(\mathrm{~d}, J=6.3 \mathrm{~Hz}, 0 \mathrm{H}), 6.32(\mathrm{ddd}, J=10.2,6.1,2.1 \mathrm{~Hz}, 1 \mathrm{H}), 5.84$ $(\mathrm{dd}, J=10.2,3.8 \mathrm{~Hz}, 1 \mathrm{H}), 4.09(\mathrm{t}, J=6.6 \mathrm{~Hz}, 2 \mathrm{H}), 3.81(\mathrm{ddd}, J=3.8$, $2.1,2.1 \mathrm{~Hz}, 1 \mathrm{H}), 1.85(\mathrm{p}, J=6.6 \mathrm{~Hz}, 2 \mathrm{H}), 1.52-1.49(\mathrm{~m}, 2 \mathrm{H}), 1.41-$ $1.20(\mathrm{~m}, 10 \mathrm{H}), 0.89(\mathrm{t}, J=6.6 \mathrm{~Hz}, 3 \mathrm{H}) \mathrm{ppm} .{ }^{13} \mathrm{C}$ NMR $(125 \mathrm{MHz}$, $\left.\mathrm{CDCl}_{3}\right): \delta=149.2(\mathrm{dd}, J=250.6,11.2 \mathrm{~Hz}), 148.6(\mathrm{dd}, J=8.2,3.0$ $\mathrm{Hz}), 142.0(\mathrm{dd}, J=247.9,14.9 \mathrm{~Hz}), 139.8,138.8,136.8(\mathrm{dd}, J=2.4$, $1.5 \mathrm{~Hz}), 132.6,131.1$ (d, $J=8.5 \mathrm{~Hz}), 129.7,129.6(\mathrm{~d}, J=3.3 \mathrm{~Hz})$, 127.8, 126.5, 123.6 (apparent t, $J=4.1 \mathrm{~Hz}$ ), 121.7 (d, $J=10.6 \mathrm{~Hz}$ ), 121.3, 119.6, 115.4, 112.9, 109.8 (dd, $J=3.3,1.2 \mathrm{~Hz}$ ), 70.1, 51.3, 45.3, 32.0, 29.5, 29.4, 29.3, 26.0, 22.8, 14.3 ppm. HRMS (MALDI + ve) calcd for $\mathrm{C}_{32} \mathrm{H}_{30} \mathrm{~F}_{2} \mathrm{~N}_{2} \mathrm{ONa}\left[(\mathrm{M}+\mathrm{Na})^{+}\right]: m / z=519.2218$, found $m / z=519.2217$. Analysis calcd (\%) for $\mathrm{C}_{32} \mathrm{H}_{30} \mathrm{~F}_{2} \mathrm{~N}_{2} \mathrm{O}$ (496.60): $\mathrm{C}$ 77.40, H 6.09, N 5.64; found: C 77.59, H 5.88, N 5.70.

2-(2,3-Difluoro-4-(octyloxy)phenyl)azulene-1,1(8aH)-dicarbonitrile (3h). To a mixture of $13 \mathrm{~h}(6.58 \mathrm{~g}, 19.8 \mathrm{mmol})$ and $\left[\mathrm{C}_{7} \mathrm{H}_{7}\right] \mathrm{BF}_{4}$ (3.62 g, $20.4 \mathrm{mmol})$ in $\mathrm{CH}_{2} \mathrm{Cl}_{2}(250 \mathrm{~mL})$ at $-78{ }^{\circ} \mathrm{C}$ under an argon atmosphere, was added $\mathrm{NEt}_{3}(2.8 \mathrm{~mL}, 20.4 \mathrm{mmol})$ over 30 $\mathrm{min}$, and the reaction mixture stirred at $-78{ }^{\circ} \mathrm{C}$ for $2 \mathrm{~h}$. Additional $\mathrm{NEt}_{3}(0.5 \mathrm{~mL}, 3.58 \mathrm{mmol})$ was added and the reaction mixture was allowed to stir for a further $60 \mathrm{~min}$. The reaction was quenched cold with $\mathrm{HCl}(50 \mathrm{~mL}, 2 \mathrm{M})$ and allowed to reach rt and diluted with water $(50 \mathrm{~mL})$. The phases were separated and the organic phase was washed with water $(250 \mathrm{~mL})$, dried over $\mathrm{MgSO}_{4}$, filtered and the solvent removed under reduced pressure. The residue was taken up in $\mathrm{CH}_{2} \mathrm{ClCH}_{2} \mathrm{Cl}(125 \mathrm{~mL})$ and treated with $\left[\mathrm{Ph}_{3} \mathrm{C}\right] \mathrm{BF}_{4}(7.96 \mathrm{~g}, 24.1 \mathrm{mmol})$ and the vessel subjected to reflux for $2 \mathrm{~h}$ under argon. The reaction mixture was cooled to $0{ }^{\circ} \mathrm{C}$ and diluted with toluene $(125 \mathrm{~mL})$ and $\mathrm{NEt}_{3}$ (3.4 mL, $24.4 \mathrm{mmol}$ ) was added. The mixture was stirred overnight, allowing the vessel to reach rt. The solvent was removed in vacuo and the residue was subjected to flash chromatography (30-50\% $\mathrm{CH}_{2} \mathrm{Cl}_{2} /$ heptane) to give $3 \mathbf{h}(2.528 \mathrm{~g}, 30 \%)$ as a yellow solid. $R_{\mathrm{f}}=0.60\left(50 \% \mathrm{CH}_{2} \mathrm{Cl}_{2} /\right.$ heptane $) . \mathrm{Mp}=95.0-97.9$ ${ }^{\circ} \mathrm{C} .{ }^{1} \mathrm{H}$ NMR $\left(500 \mathrm{MHz}, \mathrm{CDCl}_{3}\right): \delta=7.52(\mathrm{td}, J=8.5,2.0 \mathrm{~Hz}, 1 \mathrm{H})$, $7.04(\mathrm{~s}, 1 \mathrm{H}), 6.92-6.81(\mathrm{~m}, 1 \mathrm{H}), 6.57(\mathrm{dd}, J=11.3,6.4 \mathrm{~Hz}, 1 \mathrm{H})$, $6.48(\mathrm{dd}, J=11.3,6.1 \mathrm{~Hz}, 1 \mathrm{H}), 6.37(\mathrm{~d}, J=6.4 \mathrm{~Hz}, 1 \mathrm{H}), 6.31$ (ddd, $J=10.3,6.1,1.9 \mathrm{~Hz}, 1 \mathrm{H}), 5.80(\mathrm{dd}, J=10.3,3.8 \mathrm{~Hz}, 1 \mathrm{H})$, $4.10(\mathrm{t}, J=6.6 \mathrm{~Hz}, 2 \mathrm{H}), 3.74(\mathrm{ddd}, J=3.8,1.9,1.9 \mathrm{~Hz}, 1 \mathrm{H}), 1.88-$ $1.82(\mathrm{~m}, 2 \mathrm{H}), 1.51-1.45(\mathrm{~m}, 2 \mathrm{H}), 1.40-1.25(\mathrm{~m}, 8 \mathrm{H}), 0.90(\mathrm{t}, J=$ $6.8 \mathrm{~Hz}, 3 \mathrm{H}) \mathrm{ppm} .{ }^{13} \mathrm{C}$ NMR $\left(125 \mathrm{MHz}, \mathrm{CDCl}_{3}\right): \delta=150.51(\mathrm{dd}, J$ $=254.2,11.8 \mathrm{~Hz}), 149.89(\mathrm{dd}, J=8.2,3.6 \mathrm{~Hz}), 142.05(\mathrm{dd}, J=$ 249.0, $14.8 \mathrm{~Hz}$ ), 139.27, 135.90 (d, $J=14.9 \mathrm{~Hz}$ ), 132.86 (dd. $J=$ $4.3,3.3 \mathrm{~Hz}$ ), 131.08 (d, $J=1.6 \mathrm{~Hz}), 127.91,121.71(\mathrm{dd}, J=4.3$, $3.6 \mathrm{~Hz}), 121.67,119.45,115.22,112.95(\mathrm{~d}, J=9.1 \mathrm{~Hz}), 112.83$, $109.34(\mathrm{dd}, J=3.0,1.2 \mathrm{~Hz}$ ), 70.03, 50.36, 46.32, 31.91, 29.38, 29.31, 29.09, 25.95, 22.78, $14.22 \mathrm{ppm}, 1 \mathrm{C}$ masked. HRMS (MALDI +ve) calcd for $\mathrm{C}_{26} \mathrm{H}_{26} \mathrm{~F}_{2} \mathrm{~N}_{2} \mathrm{O}\left[(\mathrm{M})^{+}\right]: \mathrm{m} / z=420.2008$; $\exp$ 420.2008; analysis calcd (\%) for $\mathrm{C}_{26} \mathrm{H}_{26} \mathrm{~F}_{2} \mathrm{~N}_{2} \mathrm{O}$ (420.20): $\mathrm{C} 74.26$, H 6.23, N 6.66; found: C 74.14, H 6.35, N 6.54.

2-(4-Octylphenyl)azulene-1,1-(8aH)-dicarbonitrile (3i). To a mixture of $13 \mathbf{i}(10.01 \mathrm{~g}, 35.70 \mathrm{mmol})$ and $\left[\mathrm{C}_{7} \mathrm{H}_{7}\right] \mathrm{BF}_{4}(6.35 \mathrm{~g}$, $35.7 \mathrm{mmol})$ in $\mathrm{CH}_{2} \mathrm{Cl}_{2}(250 \mathrm{~mL})$, at $-78{ }^{\circ} \mathrm{C}$ under an argon atmosphere, was added $\mathrm{NEt}_{3}(5 \mathrm{~mL}, 35.9 \mathrm{mmol})$ over $30 \mathrm{~min}$, and the resulting mixture allowed to stir at $-78^{\circ} \mathrm{C}$ for $2 \mathrm{~h}$. Saturated aqueous $\mathrm{NH}_{4} \mathrm{Cl}(100 \mathrm{~mL})$ and water $(100 \mathrm{~mL})$ were added to the vessel and the contents allowed to reach ambient temperature. The phases were separated and the organic phase was washed with water $(200 \mathrm{~mL})$ and brine $(200 \mathrm{~mL})$. The organic phase was dried over $\mathrm{MgSO}_{4}$, filtered, and the removal of the solvent gave the crude mixture $(13.08 \mathrm{~g})$. Of this crude material $(12.84 \mathrm{~g}, 34.65 \mathrm{mmol})$ was dissolved in $\mathrm{ClCH}_{2} \mathrm{CH}_{2} \mathrm{Cl}$ $(200 \mathrm{~mL})$ and treated with $\left[\mathrm{Ph}_{3} \mathrm{C}\right] \mathrm{BF}_{4}(13.73 \mathrm{~g}, 41.58 \mathrm{mmol})$, whilst being subjected to reflux for $2 \mathrm{~h}$ under argon. After cooling the vessel, toluene $(200 \mathrm{~mL})$ was added and the vessel placed in an ice bath, where $\mathrm{NEt}_{3}(6.3 \mathrm{~mL}, 45 \mathrm{mmol})$ was added and the mixture was stirred overnight, whilst being allowed to reach rt. Aqueous $\mathrm{NH}_{4} \mathrm{Cl}(100 \mathrm{~mL})$ was added to the vessel and the phases separated. The organic phase was washed with water 
$(100 \mathrm{~mL})$ and brine $(100 \mathrm{~mL})$, dried with $\mathrm{MgSO}_{4}$, filtered and absorbed onto Celite. The material was purified by dry column vacuum chromatography (gradient elution from heptane to toluene in $10 \%$ steps) followed by recrystallization from boiling ethanol to give $3 \mathbf{i}(1.73 \mathrm{~g}, 14 \%)$. The material was stored in the freezer, as it decomposes at ambient temperature. $R_{\mathrm{f}}=0.65$ (50\% toluene/heptane). $\mathrm{Mp}=54.4-58.6{ }^{\circ} \mathrm{C} .{ }^{1} \mathrm{H} \mathrm{NMR}(500 \mathrm{MHz}$, $\left.\mathrm{CDCl}_{3}\right): \delta=7.58(\mathrm{~d}, J=8.4 \mathrm{~Hz}, 2 \mathrm{H}), 7.20(\mathrm{~d}, J=8.4 \mathrm{~Hz}, 2 \mathrm{H}), 6.77$ $(\mathrm{s}, 1 \mathrm{H}), 6.49(\mathrm{dd}, J=11.2,6.4 \mathrm{~Hz}, 1 \mathrm{H}), 6.39(\mathrm{dd}, J=11.2,6.1 \mathrm{~Hz}$, 1H), 6.31-6.05 (m, 2H), 5.75 (dd, $J=10.3,3.8 \mathrm{~Hz}, 1 \mathrm{H}$ ), 3.71 (ddd, $J=3.8,1.9,1.9 \mathrm{~Hz}, 1 \mathrm{H}), 2.57(\mathrm{t}, J=7.7 \mathrm{~Hz}, 2 \mathrm{H}), 1.62-1.52(\mathrm{~m}$, 2H), 1.34-1.01 (m, 10H), $0.81(\mathrm{t}, J=7.0 \mathrm{~Hz}, 3 \mathrm{H}) \mathrm{ppm} .{ }^{13} \mathrm{C} \mathrm{NMR}$ $\left(125 \mathrm{MHz}, \mathrm{CDCl}_{3}\right): \delta=145.7,140.5,139.1,131.4,131.1,130.7$, $129.5,128.0,127.8,126.3$, 120.6, 119.6, 115.4, 113.0, 51.3, 45.3, 36.0, 32.0, 31.3 , 29.6, 29.5, 29.4, 22.8, 14.3 ppm. HRMS (MALDI +ve) calcd for $\mathrm{C}_{26} \mathrm{H}_{27} \mathrm{~N}_{2}\left[(\mathrm{M}-\mathrm{H})^{+}\right]$: $m / z=367.2169$; $\exp$ 367.2169; analysis calcd (\%) for $\mathrm{C}_{26} \mathrm{H}_{28} \mathrm{~N}_{2}$ (368.52): $\mathrm{C} \mathrm{84.74,} \mathrm{H}$ 7.66, N 7.60; found: C 84.69, H 7.58, N 7.60.

General procedure for $14 a-e$. To a solution of 7 (either a-e) in DMSO $(20 \mathrm{~mL})$, under an argon atmosphere, was added $\mathrm{NaN}_{3}$ (1.5-2 molar equivalents) and the contents of the reaction vessel were heated to $50{ }^{\circ} \mathrm{C}$ for $2 \mathrm{~h}$. The cooled reaction mixture was poured into ice-water $(\mathrm{ca} .50 \mathrm{~g})$ and extracted with $\mathrm{Et}_{2} \mathrm{O}(3 \times 75$ $\mathrm{mL})$. The combined organics were washed with water $(100 \mathrm{~mL})$, dried over $\mathrm{MgSO}_{4}$, filtered and the volatiles removed in vacuo. Purification by flash column chromatography (1\% EtOAc/ toluene) gave $\mathbf{1 4}$ (either a-e) as a white solid.

4-((5-Azidopentyl)oxy)benzonitrile (14a). $R_{\mathrm{f}}=0.41 . \mathrm{Mp}=29.1-$ $30.5{ }^{\circ} \mathrm{C} .{ }^{1} \mathrm{H}$ NMR $\left(500 \mathrm{MHz}, \mathrm{CDCl}_{3}\right): \delta=7.57(\mathrm{~d}, J=7.6 \mathrm{~Hz}, 2 \mathrm{H})$, $6.93(\mathrm{~d}, J=7.6 \mathrm{~Hz}, 2 \mathrm{H}), 4.01(\mathrm{t}, J=6.3 \mathrm{~Hz}, 2 \mathrm{H}), 3.32(\mathrm{t}, J=6.7 \mathrm{~Hz}$, $2 \mathrm{H}), 1.84$ (p, $J=6.3 \mathrm{~Hz}, 2 \mathrm{H}), 1.68$ (p, $J=6.7 \mathrm{~Hz}, 2 \mathrm{H}), 1.65-1.51$ $(\mathrm{m}, 2 \mathrm{H}) \mathrm{ppm} .{ }^{13} \mathrm{C} \mathrm{NMR}\left(125 \mathrm{MHz}, \mathrm{CDCl}_{3}\right): \delta=162.4,134.1$, 119.4, 115.3, 104.0, 68.1, 51.4, 28.7, 28.7, 23.5 ppm. HRMS (ESP + ve) calcd for $\mathrm{C}_{12} \mathrm{H}_{14} \mathrm{~N}_{4} \mathrm{ONa}\left([\mathrm{M}+\mathrm{Na}]^{+}\right): m / z=231.1060$; $\exp$ 253.1060.

4-((6-Azidohexyl)oxy)benzonitrile $(\mathbf{1 4 b}) . R_{\mathrm{f}}=0.42 . \mathrm{Mp}=37.4-$ $40.5{ }^{\circ} \mathrm{C} .{ }^{1} \mathrm{H}$ NMR $\left(500 \mathrm{MHz}, \mathrm{CDCl}_{3}\right): \delta=7.57(\mathrm{~d}, J=8.8 \mathrm{~Hz}, 2 \mathrm{H})$, $6.93(\mathrm{~d}, J=8.8 \mathrm{~Hz}, 2 \mathrm{H}), 4.00(\mathrm{t}, J=6.4 \mathrm{~Hz}, 2 \mathrm{H}), 3.29(\mathrm{t}, J=6.8 \mathrm{~Hz}$, $2 \mathrm{H}), 1.82$ (p, $J=6.4 \mathrm{~Hz}, 2 \mathrm{H}), 1.64$ (p, $J=6.8 \mathrm{~Hz}, 2 \mathrm{H}), 1.57-1.42$ (m, 4H) ppm. ${ }^{13} \mathrm{C}$ NMR (125 MHz, $\left.\mathrm{CDCl}_{3}\right): \delta=162.5,134.1$, 119.4, 115.3, 103.9, 68.3, 51.5, 29.0, 28.9, 26.6, 25.7 ppm. HRMS $(\mathrm{ESP}+\mathrm{ve})$ calcd for $\mathrm{C}_{13} \mathrm{H}_{16} \mathrm{~N}_{4} \mathrm{ONa}\left([\mathrm{M}+\mathrm{Na}]^{+}\right): m / z=267.1217$; $\exp 267.1216$.

4-((8-Azidooctyl)oxy)benzonitrile (14c). $R_{\mathrm{f}}=0.44 . \mathrm{Mp}=33.9-$ $36.5{ }^{\circ} \mathrm{C} .{ }^{1} \mathrm{H}$ NMR $\left(500 \mathrm{MHz}, \mathrm{CDCl}_{3}\right): \delta=7.57(\mathrm{~d}, J=8.8 \mathrm{~Hz}, 2 \mathrm{H})$, $6.93(\mathrm{~d}, J=8.8 \mathrm{~Hz}, 2 \mathrm{H}), 3.99(\mathrm{t}, J=6.5 \mathrm{~Hz}, 2 \mathrm{H}), 3.26(\mathrm{t}, J=6.6 \mathrm{~Hz}$, $2 \mathrm{H}), 1.80$ (p, $J=6.5 \mathrm{~Hz}, 2 \mathrm{H}), 1.63-1.56(\mathrm{~m}, 2 \mathrm{H}), 1.53-1.16(\mathrm{~m}$, $8 \mathrm{H}) \mathrm{ppm} .{ }^{13} \mathrm{C}$ NMR $\left(125 \mathrm{MHz}, \mathrm{CDCl}_{3}\right): \delta=162.5,134.1,119.4$, 115.3, 103.8, 68.5, 51.6, 29.3, 29.2, 29.1, 28.9, 26.8, 26.0 ppm. HRMS (ESP +ve) calcd for $\mathrm{C}_{15} \mathrm{H}_{20} \mathrm{~N}_{4} \mathrm{ONa}\left([\mathrm{M}+\mathrm{Na}]^{+}\right): m / z=$ 295.1529; $\exp 295.1530$.

4-((9-Azidononyl)oxy)benzonitrile (14d). $R_{\mathrm{f}}=0.49 . \mathrm{Mp}=42.5-$ $43.2{ }^{\circ} \mathrm{C} .{ }^{1} \mathrm{H}$ NMR $\left(500 \mathrm{MHz}, \mathrm{CDCl}_{3}\right): \delta=7.57(\mathrm{~d}, J=8.8 \mathrm{~Hz}, 2 \mathrm{H})$, $6.93(\mathrm{~d}, J=8.8 \mathrm{~Hz}, 2 \mathrm{H}), 3.99(\mathrm{t}, J=6.56 \mathrm{~Hz}, 2 \mathrm{H}), 3.26(\mathrm{t}, J=6.8$ $\mathrm{Hz}, 2 \mathrm{H}), 1.79$ (t, $J=6.56 \mathrm{~Hz}, 2 \mathrm{H}), 1.60$ (t, $J=6.8 \mathrm{~Hz}, 2 \mathrm{H}), 1.51-$ $1.25(\mathrm{~m}, 10 \mathrm{H}) \mathrm{ppm} .{ }^{13} \mathrm{C} \mathrm{NMR}\left(125 \mathrm{MHz}, \mathrm{CDCl}_{3}\right): \delta=162.6$, 134.1, 119.5, 115.3, 103.8, 68.5, 51.6, 29.5, 29.3, 29.2, 29.1, 29.0,
26.8, 26.0 ppm. HRMS (ESP +ve) calcd for $\mathrm{C}_{16} \mathrm{H}_{22} \mathrm{~N}_{4} \mathrm{ONa}([\mathrm{M}+$ $\left.\mathrm{Na}]^{+}\right): m / z=309.1687$; $\exp 309.1686$.

4-((10-Azidodecyl)oxy)benzonitrile (14e). $R_{\mathrm{f}}=0.49 . \mathrm{Mp}=46.7-$ $47.7{ }^{\circ} \mathrm{C} .{ }^{1} \mathrm{H}$ NMR $\left(500 \mathrm{MHz}, \mathrm{CDCl}_{3}\right): \delta=7.57(\mathrm{~d}, J=8.9 \mathrm{~Hz}, 2 \mathrm{H})$, $6.93(\mathrm{~d}, J=8.9 \mathrm{~Hz}, 2 \mathrm{H}), 3.99(\mathrm{t}, J=6.6 \mathrm{~Hz}, 2 \mathrm{H}), 3.26(\mathrm{t}, J=6.9 \mathrm{~Hz}$, $2 \mathrm{H}), 1.80$ (p, $J=6.6 \mathrm{~Hz}, 2 \mathrm{H}), 1.60$ (p, $J=6.9 \mathrm{~Hz}, 2 \mathrm{H}), 1.48-1.31$ $(\mathrm{m}, 12 \mathrm{H}) \mathrm{ppm} .{ }^{13} \mathrm{C} \mathrm{NMR}\left(125 \mathrm{MHz}, \mathrm{CDCl}_{3}\right): \delta=162.4,134.0$, 119.3, 115.2, 103.7, 68.4, 51.5, 29.4, 29.4, 29.3, 29.1, 29.0, 28.8, 26.7, 25.9 ppm. HRMS (ESP +ve) calcd for $\mathrm{C}_{17} \mathrm{H}_{24} \mathrm{~N}_{4} \mathrm{ONa}([\mathrm{M}+$ $\left.\mathrm{Na}]^{+}\right): m / z=323.1842 ; \exp 323.1842$.

1,1-Dicyano-2-(4-(1-(5-(4-cyanophenoxy)pentyl)-1H-1,2,3-triazol4-yl)phenyl)-1,8a-dihydroazulene (4a). To a degassed solution of 15 (51 mg, $0.182 \mathrm{mmol}$ ), 14a (96 mg, $0.417 \mathrm{mmol}$ ) and Hünig's base (10 drops) in toluene $(4 \mathrm{~mL})$ was added $\mathrm{CuI}(8 \mathrm{mg}, 0.021$ $\mathrm{mmol}$ ) and the contents stirred for 2 days at rt. The solvent was removed under reduced pressure and the residue purified by flash column chromatography $\left(10 \% \mathrm{EtOAc} / \mathrm{CH}_{2} \mathrm{Cl}_{2}\right)$ to furnish 4a as a yellow solid (91 mg, 98\%). $R_{\mathrm{f}}=0.60$ (5\% EtOAc/ $\mathrm{CH}_{2} \mathrm{Cl}_{2}$ ). $\mathrm{Mp}=183.6-185.5 ; 186.9-188.7{ }^{\circ} \mathrm{C} .{ }^{1} \mathrm{H}$ NMR (500 MHz, $\left.\mathrm{CDCl}_{3}\right)$ : $\delta=7.92(\mathrm{~d}, J=8.4 \mathrm{~Hz}, 2 \mathrm{H}), 7.82(\mathrm{~s}, 1 \mathrm{H}), 7.80(\mathrm{~d}, J=8.4 \mathrm{~Hz}, 2 \mathrm{H})$, $7.56(\mathrm{~d}, J=8.8 \mathrm{~Hz}, 2 \mathrm{H}), 6.93(\mathrm{~s}, 1 \mathrm{H}), 6.90(\mathrm{~d}, J=8.8 \mathrm{~Hz}, 2 \mathrm{H})$, $6.57(\mathrm{dd}, J=11.3,6.4 \mathrm{~Hz}, 1 \mathrm{H}), 6.48(\mathrm{dd}, J=11.3,6.1 \mathrm{~Hz}, 1 \mathrm{H})$, $6.36(\mathrm{~d}, J=6.4 \mathrm{~Hz}, 1 \mathrm{H}), 6.31$ (ddd, $J=10.2,6.1,1.9 \mathrm{~Hz}, 1 \mathrm{H})$, $5.82(\mathrm{dd}, J=10.2,3.7 \mathrm{~Hz}, 1 \mathrm{H}), 4.46(\mathrm{t}, J=7.0 \mathrm{~Hz}, 2 \mathrm{H}), 3.99(\mathrm{t}$, $J=6.2 \mathrm{~Hz}, 2 \mathrm{H}), 3.80(\mathrm{ddd}, J=3.7,1.9,1.9 \mathrm{~Hz}, 1 \mathrm{H}), 2.06(\mathrm{p}, J=$ $7.0 \mathrm{~Hz}, 2 \mathrm{H}), 1.87$ (p, $J=6.2 \mathrm{~Hz}, 2 \mathrm{H}), 1.59-1.53(\mathrm{~m}, 2 \mathrm{H}) \mathrm{ppm} .{ }^{13} \mathrm{C}$ NMR (125 MHz, $\mathrm{CDCl}_{3}$ ): $\delta=162.3,146.9,139.7,138.8,134.1$, $132.5,132.2$, 131.1, 131.1, 130.2, 127.8, 126.9, 126.4, 121.3, 120.2, 119.5, 119.4, 115.3, 112.9, 104.1, 51.2, 50.4, 45.2, 30.1, 28.5, $23.2 \mathrm{ppm}$. HRMS (MALDI +ve) calcd for $\mathrm{C}_{32} \mathrm{H}_{27} \mathrm{~N}_{6} \mathrm{O}([\mathrm{M}+$ $\left.\mathrm{H}^{+}\right): m / z=511.2244$; $\exp 511.2244$. Analysis calcd (\%) for $\mathrm{C}_{32} \mathrm{H}_{26} \mathrm{~N}_{6} \mathrm{O}$ (510.60): C 75.27, $\mathrm{H}$ 5.13, $\mathrm{N}$ 16.46; found: $\mathrm{C} 75.27$, $\mathrm{H} 4.78, \mathrm{~N} 16.26$.

1,1-Dicyano-2-(4-(1-(6-(4-cyanophenoxy)hexyl)-1H-1,2,3-triazol4-yl)phenyl)-1,8a-dihydroazulene (4b). To a degassed solution of 15 (104 mg, $0.371 \mathrm{mmol}), 14 \mathrm{~b}(117 \mathrm{mg}, 0.478 \mathrm{mmol})$ and Hünig's base (10 drops) in toluene $(4 \mathrm{~mL})$ was added CuI (10 $\mathrm{mg}, 0.053 \mathrm{mmol}$ ) and the contents stirred for 2 days at rt. The solvent was removed under reduced pressure and the residue was subjected to flash column chromatography (10\% EtOAc/ $\left.\mathrm{CH}_{2} \mathrm{Cl}_{2}\right)$ and subsequently crystallized $\left(\mathrm{CH}_{2} \mathrm{Cl}_{2} /\right.$ heptane $)$ to furnish $4 \mathrm{~b}(141 \mathrm{mg}, 72 \%)$ as a yellow solid. $R_{\mathrm{f}}=0.66(5 \% \mathrm{EtOAc} /$ $\left.\mathrm{CH}_{2} \mathrm{Cl}_{2}\right) \cdot \mathrm{Mp}=54.0-55.7{ }^{\circ} \mathrm{C} .{ }^{1} \mathrm{H}$ NMR $\left(500 \mathrm{MHz}, \mathrm{CDCl}_{3}\right): \delta=$ $7.92(\mathrm{~d}, J=8.6 \mathrm{~Hz}, 2 \mathrm{H}), 7.82(\mathrm{~s}, 1 \mathrm{H}), 7.80(\mathrm{~d}, J=8.6 \mathrm{~Hz}, 2 \mathrm{H}), 7.55$ $(\mathrm{d}, J=8.9 \mathrm{~Hz}, 2 \mathrm{H}), 6.94(\mathrm{~s}, 1 \mathrm{H}), 6.91(\mathrm{~d}, J=8.9 \mathrm{~Hz}, 2 \mathrm{H}), 6.58$ (dd, $J=11.2,6.5 \mathrm{~Hz}, 1 \mathrm{H}), 6.48(\mathrm{dd}, J=11.2,6.1 \mathrm{~Hz}, 1 \mathrm{H}), 6.36(\mathrm{~d}, J=$ $6.5 \mathrm{~Hz}, 1 \mathrm{H}), 6.31$ (ddd, $J=10.2,6.1,2.1 \mathrm{~Hz}, 1 \mathrm{H}), 5.83(\mathrm{dd}, J=$ $10.2,3.8 \mathrm{~Hz}, 1 \mathrm{H}), 4.44(\mathrm{t}, J=7.1 \mathrm{~Hz}, 2 \mathrm{H}), 3.98(\mathrm{t}, J=6.3 \mathrm{~Hz}, 2 \mathrm{H})$, 3.80 (ddd, $J=3.8,2.1,2.1 \mathrm{~Hz}, 1 \mathrm{H}), 2.01$ (p, $J=7.1 \mathrm{~Hz}, 2 \mathrm{H}), 1.85-$ 1.77 (m, 2H), $1.58-1.50(\mathrm{~m}, 2 \mathrm{H}), 1.48-1.40(\mathrm{~m}, 2 \mathrm{H}) \mathrm{ppm} .{ }^{13} \mathrm{C}$ NMR (125 MHz, $\left.\mathrm{CDCl}_{3}\right): \delta=162.4,146.9,139.7,138.8,134.1$, $132.5,132.3,131.1,131.1,130.2$, 127.8, 126.9, 126.4, 121.3, 120.1, 119.6, 119.4, 115.3, 112.9, 103.9, 68.1, 51.3, 50.5, 45.3, 30.3, 28.9, 26.3, 25.6 ppm. MS (ESP +ve): $m / z=525\left([\mathrm{M}+\mathrm{H}]^{+}\right)$. Analysis calcd (\%) for $\mathrm{C}_{33} \mathrm{H}_{28} \mathrm{~N}_{6} \mathrm{O}$ (524.63): $\mathrm{C} 75.55, \mathrm{H} 5.38, \mathrm{~N}$ 16.02; found: C 75.57, H 5.39, N 16.00. 
1,1-Dicyano-2-(4-(1-(8-(4-cyanophenoxy)octyl)-1H-1,2,3-triazol4-yl)phenyl)-1,8a-dihydroazulene (4c). To a degassed solution of 15 (66 mg, $0.235 \mathrm{mmol}$ ), 14c (87 mg, $0.318 \mathrm{mmol}$ ) and Hünig's base (10 drops) in toluene $(4 \mathrm{~mL})$ was added CuI $(7 \mathrm{mg}, 0.037$ $\mathrm{mmol}$ ) and the contents stirred for 2 days at rt. The solvent was removed under reduced pressure and the residue purified by flash column chromatography $\left(10 \% \mathrm{EtOAc} / \mathrm{CH}_{2} \mathrm{Cl}_{2}\right)$ to furnish 4c $(65 \mathrm{mg}, 50 \%)$ as a yellow solid. $R_{\mathrm{f}}=0.75\left(5 \% \mathrm{EtOAc} / \mathrm{CH}_{2} \mathrm{Cl}_{2}\right)$. $\mathrm{Mp}=148.6-152.4{ }^{\circ} \mathrm{C} .{ }^{1} \mathrm{H}$ NMR $\left(500 \mathrm{MHz}, \mathrm{CDCl}_{3}\right): \delta=7.93(\mathrm{~d}, J$ $=8.5 \mathrm{~Hz}, 2 \mathrm{H}), 7.81(\mathrm{~s}, 1 \mathrm{H}), 7.79(\mathrm{~d}, J=8.5 \mathrm{~Hz}, 2 \mathrm{H}), 7.56(\mathrm{~d}, J=$ $8.8 \mathrm{~Hz}, 2 \mathrm{H}), 6.93$ (s, 1H), 6.91 (d, $J=8.8 \mathrm{~Hz}, 2 \mathrm{H}), 6.57$ (dd, $J=$ 11.3, $6.4 \mathrm{~Hz}, 1 \mathrm{H}), 6.48(\mathrm{dd}, J=11.3,6.1 \mathrm{~Hz}, 1 \mathrm{H}), 6.36(\mathrm{~d}, J=6.4$ $\mathrm{Hz}, 1 \mathrm{H}$ ), 6.31 (ddd, $J=10.2,6.1,2.0 \mathrm{~Hz}, 1 \mathrm{H}), 5.83$ (dd, $J=10.2$, $3.8 \mathrm{~Hz}, 1 \mathrm{H}), 4.42(\mathrm{t}, J=7.1 \mathrm{~Hz}, 2 \mathrm{H}), 3.97(\mathrm{t}, J=6.5 \mathrm{~Hz}, 2 \mathrm{H}), 3.80$ (ddd, $J=3.8,2.0,2.0 \mathrm{~Hz}, 1 \mathrm{H}), 2.00-1.96(\mathrm{~m}, 2 \mathrm{H}), 1.81-1.75(\mathrm{~m}$, 2H), 1.53-1.32 (m, 8H) ppm. ${ }^{13} \mathrm{C}$ NMR (125 MHz, $\left.\mathrm{CDCl}_{3}\right): \delta=$ $162.5,146.8,139.7,138.78,134.1,132.5,132.3,131.1,130.1$, $127.8,126.9,126.4,121.3,120.1,119.6,119.4,115.3,115.3$, 112.9, 103.8, 68.4, 51.2, 50.6, 45.2, 30.4, 29.2, 29.0, 29.0, 26.5, $25.9 \mathrm{ppm}, 1 \mathrm{C}$ masked. HRMS (MALDI +ve) calcd for $\mathrm{C}_{35} \mathrm{H}_{33} \mathrm{~N}_{6} \mathrm{O}$ $\left([\mathrm{M}+\mathrm{H}]^{+}\right): m / z=553.2710$; $\exp 553.2716$. Analysis calcd (\%) for $\mathrm{C}_{35} \mathrm{H}_{32} \mathrm{~N}_{6} \mathrm{O}$ (552.68): $\mathrm{C} 76.06, \mathrm{H} 5.84, \mathrm{~N} 15.21$; found: $\mathrm{C} 75.93, \mathrm{H}$ $5.88, \mathrm{~N} 15.11$.

1,1-Dicyano-2-(4-(1-(9-(4-cyanophenoxy)nonyl)-1H-1,2,3-triazol4-yl)phenyl)-1,8a-dihydroazulene (4d). To a degassed solution of 15 (49 mg, $0.175 \mathrm{mmol}$ ), 14d (49 mg, $0.179 \mathrm{mmol}$ ) and Hünig's base (10 drops) in toluene $(4 \mathrm{~mL})$ was added CuI $(7 \mathrm{mg}, 0.037$ $\mathrm{mmol}$ ) and the contents stirred for 2 days at rt. The solvent was removed under reduced pressure and the residue was subjected to flash column chromatography $\left(10 \% \mathrm{EtOAc} / \mathrm{CH}_{2} \mathrm{Cl}_{2}\right)$ followed by crystallization to give pure $\mathbf{4 d}$ (49 $\mathrm{mg}, 49 \%$ ) as a yellow solid. $R_{\mathrm{f}}=0.77$ (5\% EtOAc/ $\left.\mathrm{CH}_{2} \mathrm{Cl}_{2}\right) . \mathrm{Mp}=131.9-148.4{ }^{\circ} \mathrm{C} .{ }^{1} \mathrm{H} \mathrm{NMR}$ $\left(500 \mathrm{MHz}, \mathrm{CDCl}_{3}\right): \delta=7.94(\mathrm{~d}, J=8.4 \mathrm{~Hz}, 2 \mathrm{H}), 7.81-7.79(\mathrm{~m}$, $3 \mathrm{H}), 7.57$ (d, $J=8.77 \mathrm{~Hz}, 2 \mathrm{H}), 6.93-6.91(\mathrm{~m}, 3 \mathrm{H}), 6.58(\mathrm{dd}, J=$ 11.3, $6.3 \mathrm{~Hz}, 1 \mathrm{H}), 6.49(\mathrm{dd}, J=11.3,6.1 \mathrm{~Hz}, 1 \mathrm{H}), 6.36(\mathrm{~d}, J=6.3$ $\mathrm{Hz}, 1 \mathrm{H}$ ), 6.32 (ddd, $J=10.2,6.1,2.0 \mathrm{~Hz}, 1 \mathrm{H}), 5.83$ (dd, $J=10.2$, $3.8 \mathrm{~Hz}, 1 \mathrm{H}), 4.42(\mathrm{t}, J=7.1 \mathrm{~Hz}, 2 \mathrm{H}), 3.98(\mathrm{t}, J=6.5 \mathrm{~Hz}, 2 \mathrm{H}), 3.81$ (ddd, $J=3.8,2.0,2.0 \mathrm{~Hz}, 1 \mathrm{H}), 1.99-1.96(\mathrm{~m}, 2 \mathrm{H}), 1.78(\mathrm{p}, J=6.5$ $\mathrm{Hz}, 2 \mathrm{H}), 1.44-1.41$ (m, 2H), 1.37-1.34 (m, 8H) ppm. ${ }^{13} \mathrm{C}$ NMR $\left(125 \mathrm{MHz}, \mathrm{CDCl}_{3}\right): \delta=162.4,146.7,139.6,138.7,134.0,132.3$, $132.2,131.0,130.9,130.0,127.7,126.8,126.3$, 121.1, 119.9, 119.5, 119.3, 115.2, 112.7, 103.7, 68.3, 51.1, 50.5, 45.1, 30.3, 29.3, 29.2, 29.0, 28.9, 26.5, 25.9 ppm. HRMS (MALDI +ve) calcd for $\mathrm{C}_{36} \mathrm{H}_{35} \mathrm{~N}_{6} \mathrm{O}\left(\mathrm{M}^{+}\right): m / z=566.2789$; $\exp 566.2794$. Analysis calcd (\%) for $\mathrm{C}_{36} \mathrm{H}_{34} \mathrm{~N}_{6} \mathrm{O}$ (566.71): $\mathrm{C} 76.30, \mathrm{H} 6.05, \mathrm{~N} 14.83$; found: $\mathrm{C}$ 75.92, H 5.70, N 14.71.

1,1-Dicyano-2-(4-(1-(10-(4-cyanophenoxy)deconyl)-1H-1,2,3-triazol4-yl)phenyl)-1,8a-dihydroazulene (4e). To a degassed solution of 15 (73 mg, $0.260 \mathrm{mmol}), 14 \mathrm{e}$ (112 mg, $0.373 \mathrm{mmol}$ ) and Hünig's base (10 drops) in toluene $(4 \mathrm{~mL})$ was added CuI $(15 \mathrm{mg}, 0.079 \mathrm{mmol})$ and the contents stirred for 2 days at rt. The solvent was removed under reduced pressure and the residue was subjected to flash column chromatography $\left(10 \%\right.$ EtOAc $\left./ \mathrm{CH}_{2} \mathrm{Cl}_{2}\right)$ followed by crystallization to give pure $4 \mathrm{e}(73 \mathrm{mg}, 48 \%)$ as a yellow solid. $R_{\mathrm{f}}=0.80$ $\left(5 \%\right.$ EtOAc/ $\left.\mathrm{CH}_{2} \mathrm{Cl}_{2}\right) . \mathrm{Mp}=111.1-115.1{ }^{\circ} \mathrm{C} .{ }^{1} \mathrm{H}$ NMR $(500 \mathrm{MHz}$, $\left.\mathrm{CDCl}_{3}\right): \delta=7.94(\mathrm{~d}, J=8.4 \mathrm{~Hz}, 2 \mathrm{H}), 7.81-7.79(\mathrm{~m}, 3 \mathrm{H}), 7.56(\mathrm{~d}, J=$
$8.9 \mathrm{~Hz}, 1 \mathrm{H}), 6.93-6.91(\mathrm{~m}, 3 \mathrm{H}), 6.58(\mathrm{dd}, J=11.2,6.4 \mathrm{~Hz}, 1 \mathrm{H}), 6.49$ $(\mathrm{dd}, J=11.2,6.0 \mathrm{~Hz}, 1 \mathrm{H}), 6.36(\mathrm{~d}, J=6.4 \mathrm{~Hz}, 1 \mathrm{H}), 6.32(\mathrm{ddd}, J=$ 10.4, 6.1, $1.8 \mathrm{~Hz}, 1 \mathrm{H}), 5.83$ (dd, $J=10.4,3.7 \mathrm{~Hz}, 1 \mathrm{H}), 4.42(\mathrm{t}, J=7.0$ $\mathrm{Hz}, 2 \mathrm{H}), 3.98(\mathrm{t}, J=6.4 \mathrm{~Hz}, 2 \mathrm{H}), 3.81(\mathrm{ddd}, J=3.7,1.8,1.8 \mathrm{~Hz}, 1 \mathrm{H})$, 2.16-1.89 (m, 2H), 1.81-1.75 (m, 2H), 1.49-1.16 (m, 12H) ppm. ${ }^{13} \mathrm{C}$ $\operatorname{NMR}\left(125 \mathrm{MHz}, \mathrm{CDCl}_{3}\right): \delta=146.8,139.8,138.8,134.1,132.5,132.4$, 131.1, 131.1, 130.1, 127.9, 126.9, 126.4, 121.3, 120.1, 119.6, 119.5, 115.3, 115.3, 112.9, 103.8, 68.5, 51.3, 50.7, 45.3, 30.5, 29.5, 29.4, 29.4, 29.1, 29.1, 26.6, 26.1 ppm. HRMS (MALDI +ve) calcd for $\mathrm{C}_{37} \mathrm{H}_{36} \mathrm{~N}_{6} \mathrm{O}\left(\mathrm{M}^{+}\right): m / z=580.2945 ; \exp 580.2951$.

1,1-Dicyano-2-(4-octyl-(1H-1,2,3-triazol-4-yl)phenyl)-1,8adihydroazulene (4j). To a degassed solution of $16(98 \mathrm{mg}, 0.350$ $\mathrm{mmol}$ ), octylazide (106 mg, $0.683 \mathrm{mmol}$ ) and Hünig's base (10 drops) in toluene ( $4 \mathrm{~mL})$ was added CuI (11 mg, $0.058 \mathrm{mmol})$ and the contents stirred for 2 days at rt. The solvent was removed under reduced pressure and the residue was subjected to flash column chromatography $\left(10 \% \mathrm{EtOAc} / \mathrm{CH}_{2} \mathrm{Cl}_{2}\right)$ followed by crystallization to give pure $4 \mathbf{j}(147 \mathrm{mg}, 97 \%)$ as a yellow solid. $R_{\mathrm{f}}=0.84$ (5\% EtOAc/ $\left.\mathrm{CH}_{2} \mathrm{Cl}_{2}\right) . \mathrm{Mp}=126.1-130.6{ }^{\circ} \mathrm{C} .{ }^{1} \mathrm{H} \mathrm{NMR}$ $\left(500 \mathrm{MHz}, \mathrm{CDCl}_{3}\right): \delta=7.94(\mathrm{~d}, J=8.4 \mathrm{~Hz}, 2 \mathrm{H}), 7.81(\mathrm{~s}, 1 \mathrm{H}), 7.79$ $(\mathrm{d}, J=8.4 \mathrm{~Hz}, 2 \mathrm{H}), 6.93(\mathrm{~s}, 1 \mathrm{H}), 6.57(\mathrm{dd}, J=11.2,6.3 \mathrm{~Hz}, 1 \mathrm{H})$, $6.48(\mathrm{dd}, J=11.2,6.1 \mathrm{~Hz}, 1 \mathrm{H}), 6.35(\mathrm{~d}, J=6.3 \mathrm{~Hz}, 1 \mathrm{H}), 6.34-6.28$ $(\mathrm{m}, 1 \mathrm{H}), 5.83(\mathrm{dd}, J=10.2,3.6 \mathrm{~Hz}, 1 \mathrm{H}), 4.41(\mathrm{t}, J=7.2 \mathrm{~Hz}, 2 \mathrm{H})$, 3.80 (ddd, $J=3.7,1.9,1.9 \mathrm{~Hz}, 1 \mathrm{H}), 1.99-1.91$ (m, 2H), 1.97-1.95 (m, 2H), 1.42-1.18 (m, 8H), $0.87(\mathrm{t}, J=6.8 \mathrm{~Hz}, 3 \mathrm{H}) \mathrm{ppm} .{ }^{13} \mathrm{C}$ NMR (125 MHz, $\mathrm{CDCl}_{3}$ ): $\delta=146.6,139.7,138.7,132.3,130.9$, $130.9,129.9,127.7,126.7,126.3,121.1,120.0,119.5,115.2$, 112.7, 51.1, 50.6, 45.1, 31.7, 30.4, 29.1, 29.0, 26.5, 22.6, 14.1 ppm, 1C masked. HRMS (MALDI +ve) calcd for $\mathrm{C}_{28} \mathrm{H}_{30} \mathrm{~N}_{5}([\mathrm{M}+$ $\left.\mathrm{H}]^{+}\right): m / z=436.2496$; $\exp$ 436.2495. Analysis calcd (\%) for $\mathrm{C}_{28} \mathrm{H}_{29} \mathrm{~N}_{5}$ (435.58): C 77.21, $\mathrm{H} 6.71, \mathrm{~N} 16.08$; found: $\mathrm{C} 77.15, \mathrm{H}$ $6.80, \mathrm{~N} 15.94$.

2-(2-(Cyclohepta-2,4,6-trien-1-ylidene)-1-(4'-(octyloxy)-[1,1'biphenyl]-4-yl)ethylidene)malononitrile (17). To a stirred solution of $3 \mathbf{f}(418 \mathrm{mg}, 0.908 \mathrm{mmol})$ in $\mathrm{CH}_{2} \mathrm{Cl}_{2}(50 \mathrm{~mL})$ at $\mathrm{rt}$ under an argon atmosphere, was added $\mathrm{AlCl}_{3}$ (341 mg, 2.56 $\mathrm{mmol}$ ). After continued stirring for approximately $20 \mathrm{~min}$, TLC analysis indicated the presence of unreacted $3 \mathbf{f}$ and more $\mathrm{AlCl}_{3}$ (183 mg, $1.37 \mathrm{mmol}$ ) was added to the vessel and the reaction allowed to stir a further $20 \mathrm{~min}$. The mixture was cooled on an ice bath and quenched by addition of ice-water $(100 \mathrm{~mL})$ and the phases separated. The aqueous phase was extracted with $\mathrm{CH}_{2} \mathrm{Cl}_{2}(2 \times 50 \mathrm{~mL})$ and the combined organic phases dried over $\mathrm{MgSO}_{4}$ and filtered. The solution was diluted with heptane $(100 \mathrm{~mL})$ and the $\mathrm{CH}_{2} \mathrm{Cl}_{2}$ was removed by rotary evaporation while being kept at $0{ }^{\circ} \mathrm{C}$. The solution was placed in a freezer resulting in the crystallization of $\mathbf{1 7}$, which was isolated as a dark red solid (368 $\mathrm{mg}, 88 \%$ ). This material was stored in the freezer to prevent ring-closure back to 3f. $\mathrm{Mp}=105-109{ }^{\circ} \mathrm{C} .{ }^{1} \mathrm{H}$ NMR (500 MHz, $\mathrm{CDCl}_{3}$ ): $\delta=7.35(\mathrm{~d}, J=8.2 \mathrm{~Hz}, 2 \mathrm{H}), 7.35(\mathrm{~d}, J=8.7 \mathrm{~Hz}, 2 \mathrm{H}), 7.10(\mathrm{~d}, J$ $=8.2 \mathrm{~Hz}, 2 \mathrm{H}), 6.91(\mathrm{~d}, J=8.7 \mathrm{~Hz}, 2 \mathrm{H}), 6.12(\mathrm{~s}, 1 \mathrm{H}), 5.80(\mathrm{dd}, J$ $=11.4,1.5,1 \mathrm{H}), 5.65(\mathrm{dd}, J=12.2,2.1 \mathrm{~Hz}, 1 \mathrm{H}), 5.59-5.49(\mathrm{~m}$, $2 \mathrm{H}), 5.46-5.42(\mathrm{~m}, 1 \mathrm{H}), 5.10(\mathrm{dd}, J=12.2,7.8 \mathrm{~Hz}, 1 \mathrm{H}), 3.69(\mathrm{t}$, $J=6.5 \mathrm{~Hz}, 2 \mathrm{H}), 1.68-1.63(\mathrm{~m}, 2 \mathrm{H}), 1.39-1.34(\mathrm{~m}, 2 \mathrm{H}), 1.33-$ $1.18(\mathrm{~m}, 8 \mathrm{H}), 0.92(\mathrm{t}, J=7.0 \mathrm{~Hz}, 3 \mathrm{H}) \mathrm{ppm} .{ }^{13} \mathrm{C} \mathrm{NMR}(125 \mathrm{MHz}$, $\left.\mathrm{CDCl}_{3}\right): \delta=167.8,160.1,152.9,143.7,142.2,135.2,134.7$, 
134.2, 133.3, 133.2, 132.7, 132.1, 129.3, 128.6, 128.4, 127.6, 119.8, 115.3, 78.4, 68.1, 32.2, 29.8, 29.7, 29.7, 26.4, 23.1, 14.4 ppm, 1C masked. HRMS (MALDI +ve) calcd for $\mathrm{C}_{32} \mathrm{H}_{32} \mathrm{~N}_{2} \mathrm{ONa}$ $\left[(\mathrm{M}+\mathrm{Na})^{+}\right]: m / z=483.2407$, found $m / z=483.2412$. Analysis calcd (\%) for $\mathrm{C}_{32} \mathrm{H}_{32} \mathrm{~N}_{2} \mathrm{O}$ (496.62): C 83.49, $\mathrm{H}$ 7.00, N 6.08; found: $\mathrm{C}$ 83.38, $\mathrm{H}$ 7.11, $\mathrm{N} 6.14$.

2-(2-(Cyclohepta-2,4,6-trien-1-ylidene)-1-(2',3'-difluoro-4' -hydroxy[1,1'-biphenyl]-4-yl)ethylidene)malononitrile (18). To a stirred solution of $3 \mathrm{~g}$ (210 $\mathrm{mg}, 0.423 \mathrm{mmol})$ in $\mathrm{CH}_{2} \mathrm{Cl}_{2}(50 \mathrm{~mL})$ at $\mathrm{rt}$, under an argon atmosphere, was added $\mathrm{AlCl}_{3}(1.010 \mathrm{~g}, 7.58 \mathrm{mmol})$ and the mixture was stirred for $20 \mathrm{~min}$. TLC analysis indicated the presence of unreacted $3 \mathrm{~g}$ and more $\mathrm{AlCl}_{3}(540 \mathrm{mg}, 4.05 \mathrm{mmol}$ ) was added, whereby the reaction was allowed to stir a further $20 \mathrm{~min}$. The mixture was cooled on an ice bath and quenched by addition of ice-water $(100 \mathrm{~mL})$ after which the phases were separated. The aqueous phase was extracted with $\mathrm{CH}_{2} \mathrm{Cl}_{2}(2 \times 50 \mathrm{~mL})$ and the combined organic phases was dried with $\mathrm{MgSO}_{4}$ and filtered. Heptane was added $(100 \mathrm{~mL})$, and the solution concentrated under reduced pressure at $0{ }^{\circ} \mathrm{C}$, whereby the solution was placed at -18 ${ }^{\circ} \mathrm{C}$ for $16 \mathrm{~h}$, resulting in the crystallization of 18 (85 mg, 52\%) as a dark red solid. This material was stored in the freezer to prevent ring-closure back to $3 \mathrm{~g}$. $\mathrm{Mp}=129.7-131.0$ (color change to yellow), 168 (decomposes) ${ }^{\circ} \mathrm{C} .{ }^{1} \mathrm{H}$ NMR $\left(500 \mathrm{MHz}, \mathrm{CDCl}_{3}\right): \delta=7.61(\mathrm{~d}, J=$ $7.9 \mathrm{~Hz}, 2 \mathrm{H}), 7.47$ (d, $J=7.9 \mathrm{~Hz}, 2 \mathrm{H}), 7.13(\mathrm{td}, J=8.3,2.2 \mathrm{~Hz}, 1 \mathrm{H})$, 6.90-6.84 (m, 1H), 6.75-6.69 (m, 1H), 6.45-6.37 (m, 2H), 6.35 (s, $1 \mathrm{H}), 6.33-6.27(\mathrm{~m}, 1 \mathrm{H}), 5.94(\mathrm{~d}, J=4.6 \mathrm{~Hz}, 1 \mathrm{H}), 5.60$ (br s, $1 \mathrm{H}$, exchanges $\mathrm{D}_{2} \mathrm{O}$ ) ppm. ${ }^{13} \mathrm{C} \mathrm{NMR}\left(125 \mathrm{MHz}, \mathrm{CDCl}_{3}\right): \delta=\mathrm{ppm}^{13} \mathrm{C}$ NMR (125 MHz, $\left.\mathrm{CDCl}_{3}\right): \delta=168.4,154.1,148.4(\mathrm{dd}, J=250.4,11.4$ $\mathrm{Hz}), 145.0$ (dd, $J=11.3,2.0 \mathrm{~Hz}), 142.3,137.7$ (dd, $J=2.5,1.6 \mathrm{~Hz}$ ), 135.6, 135.3, 135.2, 134.5, 134.1, 133.7, 129.9 (d, $J=3.3 \mathrm{~Hz}), 128.6$, $124.3(\mathrm{dd}, J=3.8,3.8 \mathrm{~Hz}), 121.4(\mathrm{~d}, J=9.8 \mathrm{~Hz}), 118.9,115.1,114.6$, 112.6 (dd, $J=3.6,1.0 \mathrm{~Hz}$ ) ppm, 2Cs masked. HRMS (MALDI +ve) calcd for $\mathrm{C}_{32} \mathrm{H}_{31} \mathrm{~F}_{2} \mathrm{~N}_{2} \mathrm{O}\left[(\mathrm{M}+\mathrm{H})^{+}\right]: m / z=358.1147$, found $m / z=$ 385.1142 .

\section{Acknowledgements}

The Danish Council for Independent Research | Technology and Production Sciences (\#12-126668) and University of Copenhagen are acknowledged for financial support. Dr Theis Brock-Nannestad, University of Copenhagen, is thanked for helpful discussions.

\section{Notes and references}

1 Y. Li, A. Urbas and Q. Li, J. Am. Chem. Soc., 2012, 134, 9573. 2 T. Ikeda and O. Tsutsumi, Science, 1995, 268, 1873.

3 R. H. Berg, S. Hvilsted and P. S. Ramanujam, Nature, 1996, 383, 505.

4 A. Y. Bobrovsky, N. I. Boiko, V. P. Shibaev, M. A. Kalik and M. Krayushkin, J. Mater. Chem., 2001, 11, 2004.

5 T. J. Bunning, R. L. Crane and W. W. Adams, Adv. Mater. Opt. Electron., 1992, 1, 293.
6 S. Z. Janicki and G. B. Schuster, J. Am. Chem. Soc., 1995, 117, 8524.

7 J. Daub, T. Knöchel and A. Mannschreck, Angew. Chem., Int. Ed. Engl., 1984, 23, 960.

8 T. Li, M. Jevric, J. R. Hauptmann, R. Hviid, Z. Wei, R. Wang, N. E. Reeler, E. Thyrhaug, S. Petersen, J. A. S. Meyer, N. Bovet, T. Vosch, J. Nygård, X. Qiu, W. Hu, Y. Liu, G. C. Solomon, H. G. Kjaergaard, T. Bjørnholm, M. B. Nielsen, B. W. Laursen and K. Nørgaard, Adv. Mater., 2013, 25, 4164.

9 S. L. Broman and M. B. Nielsen, Phys. Chem. Chem. Phys., 2014, 16, 21172.

10 D. Demus, J. Goodby, G. W. Gray, H.-W. Spies and V. Vill, Handbook of liquid crystals, Fundamentals, Wiley-VCH, New York, 1998, ch 6.

11 See for example: I. M. Saez and J. W. Goodby, J. Mater. Chem., 2003, 13, 2727; A. R. E. Brás, S. Henriques, T. Casimoro, A. Aguiar-Ricardo, J. Sotomayor, J. Caldeira, C. Santos and M. Dionísio, Liq. Cryst., 2007, 34, 591; I. M. Saez and J. W. Goodby, Liq. Cryst., 2010, 26, 1101; R. J. Mandle, E. J. Davis, C.-C. A. Voll, D. J. Lewis, S. J. Cowling and J. W. Goodby, J. Mater. Chem. C, 2015, 3, 2380.

12 See for example: H. Sorkin, Mol. Cryst. Liq. Cryst., 1980, 56, 279; M. Hara, Y. Iwakabe, K. Tochigi, H. Sasabe, A. F. Garito and A. Yamada, Nature, 1990, 344, 228; H. Sugisawa, H. Toriumi and H. Watanabe, Mol. Cryst. Liq. Cryst., 1992, 214, 11.

13 A. S. Achalkumar, D. S. Shankar Rao and C. V. Yelamaggad, New J. Chem., 2014, 38, 4235.

14 S. M. Kelly, Helv. Chim. Acta, 1989, 72, 594.

15 C. W. Tornøe, C. Christensen and M. Meldal, J. Org. Chem., 2002, 67, 3057; H. C. Kolb, M. G. Finn and B. Sharpless, Angew. Chem., Int. Ed., 2001, 40, 2004.

16 R. Cristiano, D. M. P. de Oliveira Santos, G. Conte and H. Gallardo, Liq. Cryst., 2006, 33, 997.

17 H. S. Lissau, S. L. Broman, M. Jevric, A. Ø. Madsen and M. B. Nielsen, Aust. J. Chem., 2014, 67, 531.

18 C. R. Parker, C. G. Tortzen, S. L. Broman, M. SchauMagnussen, K. Kilså and M. B. Nielsen, Chem. Commun., 2011, 47, 6102.

19 S. L. Broman, M. Jevric and M. B. Nielsen, Chem.-Eur. J., 2013, 19, 9542.

20 S. Diele, S. Tosch, S. Mahnke and D. Demus, Cryst. Res. Technol., 1991, 26, 809.

21 Z. Puterová, J. Romiszewski, J. Mieczkowski and E. Gorecka, Tetrahedron, 2012, 68, 8172.

22 For a review on photoalignment techniques, see: T. Seki, S. Nagano and M. Hara, Polymer, 2013, 54, 6053.

23 L. Gobbi, P. Seiler, F. Diederich, V. Gramlich, C. Boudon, J.-P. Gisselbrecht and M. Gross, Helv. Chim. Acta, 2001, 84, 743.

24 M. Santella, V. Mazzanti, M. Jevric, C. R. Parker, S. L. Broman, A. D. Bond and M. B. Nielsen, J. Org. Chem., 2012, 77, 8922 . 